\title{
1 The PIF1-MIR408-Plantacyanin Repression Cascade Regulates Light Dependent Seed
}

\section{Germination}

3

4 Anlong Jiang ${ }^{1,2}$, Zhonglong Guo ${ }^{1}$, Jiawei Pan ${ }^{1, \#}$, Yan Zhuang ${ }^{2}$, Daqing Zuo ${ }^{1}$, Chen Hao ${ }^{1}$, Zhaoxu

$5 \mathrm{Gao}^{2}$, Peiyong $\mathrm{Xin}^{3}$, Jinfang $\mathrm{Chu}^{3}$, Shangwei Zhong ${ }^{1}$, Lei $\mathrm{Li}^{1,2, *}$

$7 \quad{ }^{1}$ State Key Laboratory of Protein and Plant Gene Research, School of Life Sciences and School

8 of Advanced Agricultural Sciences, Peking University, Beijing 100871, China

$9{ }^{2}$ Peking-Tsinghua Center for Life Sciences, Academy for Advanced Interdisciplinary Studies,

10 Peking University, Beijing 100871, China

$11{ }^{3}$ National Center for Plant Gene Research (Beijing), Institute of Genetics and Developmental

12 Biology, Chinese Academy of Sciences, Beijing 100101, China

13

14 \# Present address: Syngenta Group China, Beijing 100031, China

$15 *$ Corresponding author (lei.li@pku.edu.cn) 


\section{ABSTRACT}

17 Light-sensing seed germination is a vital process for the seed plants. A decisive event in light-

18 induced germination is degradation of the central repressor PHYTOCHROME INTERACTING

19 FACTOR1 (PIF1). It is also known that the balance between gibberellic acid (GA) and abscisic

20 acid (ABA) critically controls germination. But the cellular mechanisms linking PIF1 turnover to

21 hormonal rebalancing remain elusive. Here, employing far-red light-induced Arabidopsis seed

22 germination as the experimental system, we identified Plantacyanin (PLC) as an inhibitor of

23 germination, which is a storage vacuole-associated blue copper protein highly expressed in

24 mature seed and rapidly silenced during germination. Molecular analyses showed that PIF1

25 directly binds to the MIR408 promoter and represses miR408 accumulation, which in turn post-

26 transcriptionally modulates $P L C$ abundance, thus forming the PIF 1-MIR408-PLC repression

27 cascade for translating PIF1 turnover to PLC turnover during early germination. Genetic analysis,

28 RNA-sequencing, and hormone quantification revealed that $P L C$ is necessary and sufficient to

29 maintain the PIF1-mediated seed transcriptome and the low-GA-high-ABA state. Furthermore,

30 we found that PLC domain organization and regulation by miR408 are conserved features in

31 seed plants. These results unraveled a cellular mechanism whereby PIF1-relayed external light

32 signals are converted through PLC-based copper mobilization into internal hormonal profiles for

33 controlling seed germination.

\section{Keywords}

36 Seed germination; Gibberellic acid; Abscisic acid; Light signaling; Copper homeostasis;

37 Plantacyanin; miR408; PIF1 


\section{Introduction}

39 The seed is an embryonic plant enclosed in a protective capsule. After reaching full size, the embryo undergoes elaborate dehydration to establish a dormant state that helps the embryo withstand extreme environments and survive for long periods (Bewley 1997; Finch-Savage and Leubner-Metzger, 2006; Angelovici et al., 2010). Given the right environmental conditions, the desiccated seed germinates by taking up water and resuming embryo development, utilizing energy and nutrient stored in the seed (Bewley 1997; Finch-Savage and Leubner-Metzger, 2006; Finkelstein et al., 2008; Née et al., 2017). GA and ABA are the main plant hormones that control seed dormancy and germination. As the embryo matures, ABA is synthesized and signals the embryo to initiate the buildup of storage compounds and undergo desiccation (Nambara and Marion-Poll, 2005; Finkelstein et al., 2008; Angelovici et al., 2010; Shu et al., 2016). ABA is also important for maintaining seed dormancy and preventing precocious germination (Bewley 1997; Finch-Savage and Leubner-Metzger, 2006; Finkelstein et al., 2008). Conversely, GA is a crucial hormone to break down dormancy and promote germination (Kallioo and Piiroinen 1959; Bewley 1997; Finch-Savage and Leubner-Metzger, 2006; Yamaguchi, 2008). It has been wellestablished that the GA/ABA balance critically determines the germination capacity (Nambara and Marion-Poll, 2005; Yamaguchi, 2008; Shu et al., 2016; Née et al., 2017).

Seed monitors a wide range of environmental factors, including ambient light, for germination decision-making (Oh et al., 2004; Finch-Savage and Leubner-Metzger, 2006; Seo et al., 2009; Jiang et al., 2016). Molecular mechanisms of light perception and signaling during germination are well understood in the model plant Arabidopsis. The basic helix-loop-helix type transcription factor PIF1 is an essential negative regulator of light-dependent germination (Oh et al., 2004; Leivar and Quail, 2010; Shi et al., 2015). PIF1 is stabilized by DE-ETIOLATED 1 and other signaling molecules and thus highly accumulates in the seed kept in darkness (Oh et al., 2004; 2006; Shi et al., 2015). Under light irradiation, phytochromes are activated and enter the nucleus to interact with PIF1, thereby reducing its activity and promoting its degradation via the 26S proteasome (Oh et al., 2006; Castillon et al., 2007; Shen et al., 2008; Leivar and Quail, 2010). Rapid removal of PIF1 is critical for maintaining the light-regulated transcriptome in imbibed seed (Oh et al., 2009; Shi et al., 2013; Pfeiffer et al., 2014) and ultimately establishing

67 the high-GA-low-ABA state (Oh et al., 2006; 2007). However, extensive search has not revealed 
2009; Cho et al., 2012). Consequently, the cellular events ensued by PIF1 turnover that lead to

70 hormonal rebalancing have not been elucidated and it is not known whether these events are

71 conserved in seed plants.

A fundamental cellular process during seed germination is the mobilization of mineral nutrients sequestered in the storage vacuoles to sustain embryo growth before efficient uptake systems are established in the root (Lanquar et al., 2005; Kim et al., 2006; Roschzttardtz et al., 2009; Née et al., 2017; Paszkiewicz et al., 2017). Studies in Arabidopsis and other plants have shown that transition metals are released from vacuolar stores via tonoplast-localized transporters and then transported to various cellular destinations (Lanquar et al., 2005; Kim et al., 2006; Eroglu et al., 2017). While the physiological consequences of insufficient or mis-regulated mineral mobilization have been abundantly documented (Lanquar et al., 2005; Kim et al., 2006), whether metal mobilization contributes to hormonal profile establishment in light dependent germination is not well characterized.

Copper is an essential transition metal by serving as the cofactor for a number of cuproproteins with vital functions (Burkhead et al., 2009; Peñarrubia et al., 2015). Because free cellular copper is highly reactive and produces detrimental hydroxyl radicals, elaborate homeostasis and transport systems are present for the precise control of copper delivery to specific targets (Burkhead et al., 2009). The Arabidopsis genome encodes approximately 260 copper dependent proteins (Schulten et al., 2019). Among them, small blue copper proteins, containing a characteristic mononuclear type-I copper binding site, play important roles in redox reactions and copper homeostasis (Rydén and Hunt 1993; Guss et al., 1998; De Rienzo et al. 2000; Giri et al. 2004). Plastocyanin is the most abundant small blue copper protein in plants and

91 an indispensable electron carrier in the Z-scheme of photosynthesis (Molina-Heredia et al., 2003;

92 Weigel et al., 2003). Plants also have a specific family of blue copper proteins called

93 phytocyanins that are divided into four subfamilies, including PLCs, uclacyanins, stellacyanins,

94 and early nodulin-like proteins, based on differences in the copper binding site and domain

95 organization (Guss et al., 1998; Nersissian et al., 1998; Sun et al., 2019). Phytocyanins have been 96 widely implicated in plant development processes such as pollen tube chemotropism and nodule 97 development (Kim et al. 2003; Dong et al. 2005; Sun et al., 2019). They have also been

98 implicated in stress responses such as pathogen resistance and drought and salinity tolerance 99 (Jung and Hwang 2000; Ruan et al. 2011; Feng et al., 2013). However, their involvement in seed 
100 germination has not been investigated.

101 In this study, we focused on $P L C$ that is highly expressed in the seed with contrasting

102 expression patterns during seed development and germination. Through comprehensive

103 molecular and genetic analyses, we delineated the PIF1-MIR408-PLC repression cascade for

104 regulating PLC turnover during far-red light induced germination. We showed that PLC locates

105 to the storage vacuole and is necessary and sufficient to maintain PIF 1-mediated seed

106 transcriptome and the low-GA-high-ABA state. These results unraveled PLC-based copper

107 mobilization as a potentially conserved cellular mechanism for converting PIF1-relayed light

108 signals into hormonal profiles that control seed germination.

109 
bioRxiv preprint doi: https://doi.org/10.1101/2020.07.20.212340; this version posted July 21, 2020. The copyright holder for this preprint (which was not certified by peer review) is the author/funder. All rights reserved. No reuse allowed without permission.

110 


\section{RESULTS}

\section{PLC Exhibits Distinctive Expression Pattern in Seed Development and Germination}

113 To investigate whether the phytocyanin encoding genes are involved in seed germination, we

114 examined their expression pattern using a gene expression atlas in Arabidopsis (eFP Browser;

115 Winter et al., 2007). We found that 31 of the 37 phytocyanin genes were expressed during seed

116 formation and germination (Figure 1A). Among these, the single gene encoding PLC

117 (At2g02850) exhibited the highest expression level in mature seed (Figure 1A). PLC transcript

118 level was low in early seed development, but was drastically induced at seed stage 7,

119 progressively increased thereafter, and peaked at seed maturation (Figure 1B). Interestingly, PLC

120 was drastically silenced upon vernalization-induced germination (Figure 1B). To validate PLC

121 expression pattern in the germination phase at the protein level, we generated the $p P L C: P L C$ -

122 GFP transgenic Arabidopsis plants in which the PLC coding sequence was fused to the green

123 fluorescence protein (GFP) and driven by the native $P L C$ promoter. Immunoblotting with an

124 antibody against GFP revealed that the level of the PLC-GFP fusion protein was drastically

125 decreased $24 \mathrm{~h}$ after vernalization (Figure $1 \mathrm{C}$ ). These results indicate that PLC is highly

126 expressed in mature seed and silenced during germination.

127 To elucidate the dynamics of $P L C$ repression during light-induced germination, we

128 employed a previously described far-red light initiated, phytochrome A (phyA) dependent

129 germination assay (Oh et al., 2006; 2009; Cho et al., 2012). In the so-called phy $\mathrm{A}_{\mathrm{OFF}}$ regime of

130 this assay, imbibed seeds are exposed to brief far-red light to inactivate phyB, then kept in

131 darkness to allow inactive phyA to accumulate that does not break dormancy. In the phy $A_{\mathrm{ON}}$

132 regime, a second far-red irradiation with longer duration is used to activate phyA that induces

133 germination (Figure 1D). Using reverse transcription coupled quantitative PCR (RT-qPCR), we

134 observed that PLC transcript level remained steady during the time course of phy $\mathrm{A}_{\mathrm{OFF}}$ (Figure

135 1E). In contrast, after the second far-red irradiation in phy $A_{\mathrm{ON}}, P L C$ level was maintained only

136 until the $2 \mathrm{~h}$ time point, but drastically reduced by $4 \mathrm{~h}$, and remained low thereafter (Figure 1E).

137 To reveal subcellular localization of PLC, we transiently expressed the PLC-GFP reporter

138 in onion epidermal cells and found that the GFP signals predominantly aligned the periphery of

139 the central vacuole (Figure $1 \mathrm{~F}$ ). COPT5 is a member of the CTR-like high-affinity $\mathrm{Cu}$

140 transporters residing on the tonoplast (Klaumann et al., 2011). We found that PLC-GFP

141 colocalized with mCherry-tagged COPT5 co-expressed in the same onion epidermal cell (Figure 
142 1F), indicating that transiently expressed PLC is associated with the vacuole. To examine PLC

143 localization in the seed, we utilized the $p P L C: P L C$-GFP transgenic line. In imbibed seed kept in

144 darkness, GFP fluorescence was observed surrounding autofluorescence of storage vacuoles

145 (Figure 1G) (Paszkiewicz et al., 2017). Consistent with the expression profile of PLC (Figure

146 1E), the GFP signals disappeared after far-red irradiation (Figure 1G). These observations

147 indicate that storage vacuole-associated PLC is rapidly silenced following phyA activation.

\section{PLC Negatively Regulates Germination}

150 The expression pattern of $P L C$ inspired us to genetically investigate its role in germination. We

151 employed the previously characterized Arabidopsis mutant with an intronic T-DNA insertion

152 (Dong et al., 2005) and named this knockdown allele plc-1 (Supplemental Figure 1A). We also

153 generated a deletion mutant using the CRISPR/Cas9 system with paired guide RNAs. The

154 resulting homozygous mutant, containing a $506 \mathrm{bp}$ deletion that spans the entire coding region,

155 was named plc-2 (Supplemental Figure 1A). RT-qPCR confirmed that the plc-2 allele had

156 essentially undetectable $P L C$ transcript level in comparison to the wild type (Supplemental

157 Figure 1B). Consistent with previous characterization of plc-1 (Dong et al., 2005), we found that

158 both $p l c$ mutants exhibited no apparent difference in the appearance of mature seed compared to

159 the wild type (Supplemental Figure 1C). However, in contrast to the wild type seed that failed to

160 germinate in phy $\mathrm{A}_{\mathrm{OFF}}$, germination frequency of $p l c-1$ and $p l c-2$ significantly increased to $17.3 \%$

161 and $23.3 \%$ by $120 \mathrm{~h}$ in phy $\mathrm{A}_{\mathrm{OFF}}$, respectively (Figure $2 \mathrm{~A}$ and $2 \mathrm{~B}$ ). The approximately $40 \%$

162 germination rate of wild type seed in phy $\mathrm{A}_{\mathrm{ON}}$ was significantly elevated to $48.0 \%$ and $62.7 \%$ for

$163 p l c-1$ and $p l c-2$, respectively (Figure $2 \mathrm{~A}$ and $2 \mathrm{C}$ ). These results indicate that PLC is necessary for

164 effective inhibition of germination in both phy $\mathrm{A}_{\mathrm{OFF}}$ and phy $\mathrm{A}_{\mathrm{ON}}$.

165 Moreover, we generated the iPLC-OX transgenic Arabidopsis plants in which the

166 expression of PLC was under the control of a $\beta$-estradiol-inducible promoter (Zuo et al., 2000).

167 Mature seed of this line also exhibited no morphological difference from the wild type

168 (Supplemental Figure 1C). The PLC transcript was induced to high levels in the $i P L C-O X$ seed

169 under both phy $A_{\mathrm{OFF}}$ and phy $\mathrm{A}_{\mathrm{ON}}$ but maintained its normal pattern in the wild type after the

170 application of $\beta$-estradiol (Figure 2D; Supplemental Figure 1D). We found that $\beta$-estradiol

171 treatment significantly reduced germination rate of the $i P L C-O X$ seed, but not of the wild type,

172 in both phy $A_{\mathrm{OFF}}$ and phyA $\mathrm{ON}_{\mathrm{O}}$ (Figure $2 \mathrm{E}$ and $2 \mathrm{~F}$ ). These results indicate that $P L C$ is sufficient for 
173

inhibiting germination.

Young seedlings germinated in darkness switch to a different growth mode, termed greening, upon exposure to light (Zhong et al., 2009). Visual inspection revealed that PLC represses this process as the greening rate of $p l c-2$ (referred to as $p l c$ hereafter) was significantly higher than that of the wild type after dark-germinated seedlings were exposed to white light for $24 \mathrm{~h}$ (Figure 3A and 3B). Fluorescence spectral analysis confirmed that the levels of both chlorophylls and the precursor protochlorophyllide in plc seedlings were higher than those in the wild type (Figure 3C). Collectively, these results established PLC as a negative regulator for germination and the ensued post-germinative growth in light.

\section{PLC Is Silenced by miR408 during Germination}

In our quest of identifying upstream regulators for $P L C$, we noted that $P L C$ is a proven target of miR408 (Abdel-Ghany and Pilon, 2008; Zhang and Li, 2013). Using the standard assay of 5' RNA ligation-based amplification of cDNA ends, we reassured miR408-guided cleavage of $P L C$ mRNA in imbibed seed, which occurred between the $10^{\text {th }}$ and $11^{\text {th }}$ nucleotides in the miR408 recognition site (Figure 4A). From degradome sequencing data for young seedlings, we retrieved reads mapped to the predicted miR408 recognition site in PLC (Figure 4B), further confirming miR408 mediated cleavage of the PLC transcript. RT-qPCR analysis showed that miR408 level in the seed was stable in the phy $\mathrm{A}_{\mathrm{OFF}}$ regime but drastically elevated $2 \mathrm{~h}$ after the second far-red irradiation and peaked at $8 \mathrm{~h}$ in phy $\mathrm{A}_{\mathrm{ON}}$ (Figure 4C). Thus, miR408 and PLC exhibit reciprocal expression pattern following phyA activation (compare Figure 1E and 4C).

To test the effect of miR408 on PLC expression during germination, we employed the miR408-overexpressing line (miR408-OX) in which the enhanced Cauliflower Mosaic Virus $35 S$ promoter was used to drive miR408 expression and the miR408-silencing line (amiR408) generated by the artificial miRNA method (Zhang and Li, 2013; Zhang et al., 2014). In the miR408-OX and amiR408 seeds, expression of PLC in phy $A_{\mathrm{OFF}}$ was significantly decreased and increased in comparison to the wild type, respectively (Figure 4D). In phyA $\mathrm{ON}_{\mathrm{ON}}$, while $P L C$ level was generally lowered compared to that in phy $\mathrm{A}_{\mathrm{OFF}}$, the trend of relative $P L C$ abundance in the wild type, miR408-OX, and $a m i R 408$ seeds maintained the same (Figure 4D). Taken together, these results indicate that miR408 negatively modulates $P L C$ transcript level through the canonical transcript cleavage mechanism during early seed germination. 
miR408 Is a Positive Regulator of Germination

206 To investigate the role of miR408 in germination, we examined phenotypes of the miR408-OX

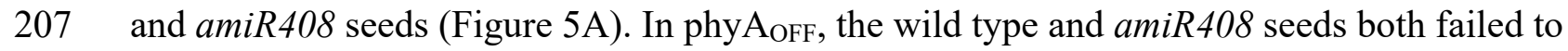

208 germinate while the germination frequency of $m i R 408-O X$ increased to about $80 \%$ over the time

209 course (Figure 5B). In phyA $\mathrm{ON}$, virtually $100 \%$ of the miR408-OX seed germinated after $120 \mathrm{~h}$

210 (Figure 5C). In contrast, the germination frequency of amiR408 only reached approximately 30\%

211 after $120 \mathrm{~h}$ in phy $_{\mathrm{ON}}$, significantly lower than that of the wild type (Figure 5C). Additionally,

212 we found that miR408 promotes the greening process as the greening rate of $m i R 408-O X$ and

213 amiR408 was significantly higher and lower than that of the wild type, respectively (Figure 5D to

$2145 \mathrm{~F}$ ). These results demonstrate miR408 as a positive regulator for germination and post-

215 germinative growth in light.

216 Because the phenotype of $m i R 408-O X$ was stronger than that of $p l c$, we examined other

217 miR408 target genes in germination. In Arabidopsis, miR408 has four validated targets that all

218 encode cuproproteins, including PLC, LACCASE 3, 12, and 13 (Abdel-Ghany and Pilon, 2008;

219 Zhang and Li, 2013; Zhang et al., 2014). Based on known expression profiles (Winter et al.,

220 2007; Zhuang et al., 2020), the three $L A C$ genes did not exhibit substantial expression in the seed

221 (Supplemental Figure 2A). Phenotypic comparison of the lac3, lac12, lac13, and plc single

222 mutants revealed that only plc displayed significantly elevated germination frequency than the

223 wild type in both phy $\mathrm{A}_{\mathrm{OFF}}$ and phy $\mathrm{A}_{\mathrm{ON}}$ (Supplemental Figure 2B). We further generated the

224 lac12 lac13 double mutant and the plc lac12 lac13 triple mutant. We found that the plc lac12

225 lac13 seed exhibited the same germination phenotype as plc while lac12 lac13 showed no

226 difference from the wild type (Supplemental Figure 2C). These results indicate that other

227 miR408 target genes are not involved in germination. Thus, whether the relatively weaker

228 phenotype of PLC loss-of-function was due to the compensation by other phytocyanins warrants

229 further investigation.

231 PIF1 Directly Suppresses MIR408 to Promote PLC Expression

232 Our next goal was to find out how the miR408-PLC module is regulated by light signaling.

233 Previously, we reported that ELONGATED HYPOCOTYL 5 (HY5), by binding to the G-box in

234 the MIR408 promoter, activates MIR408 in response to increasing light irradiation (Zhang et al., 
236 (Chen et al., 2013; Toledo-Ortiz et al., 2014; Shi et al., 2018), we investigated the possibility that

237 PIF1 transcriptionally represses MIR408. Using PIF1 chromatin immunoprecipitation (ChIP)

238 sequencing data (Pfeiffer et al., 2014), we identified a significant PIF1 binding peak in the

239 MIR408 promoter encompassing the G-box (Figure 6A). Using the PIF1-OX line expressing

240 MYC-tagged PIF1 driven by the $35 S$ promoter (Oh et al., 2004), we performed ChIP with an

241 anti-MYC antibody. qPCR analysis revealed that PIF1 occupancy at the G-box containing DNA

242 fragment was enriched over fivefold in PIF1-OX relative to the wild type (Figure 6B),

243 confirming PIF 1 as a direct upstream regulator of MIR408.

244 To ascertain the net effect of PIF1 on the MIR408 promoter, we employed the firefly

245 luciferase (LUC) and Renilla luciferase (REN) dual reporter system (Hellens et al., 2005). We

246 generated the $p M I R 408: L U C$ reporter and the 35S:PIF1 effector constructs (Figure 6C).

247 Following transfection of tobacco leaf protoplasts, we found that co-expression of PIF1

248 significantly reduced the LUC/REN ratio (Figure 6D), indicating that PIF1 negatively modulates

249 the MIR408 promoter. To corroborate this relationship in Arabidopsis, we fused the $\beta$ -

250 glucuronidase (GUS) coding region with the MIR408 promoter and expressed the same reporter

251 gene in either the wild type (pMIR408:GUS/WT) or pif1 (pMIR408:GUS/pif1) background

252 (Figure 6E). We found that GUS activity, mainly detected in the cotyledons of imbibed seed, was

253 higher in the pifl background in both phy $\mathrm{A}_{\mathrm{OFF}}$ and phy $\mathrm{A}_{\mathrm{ON}}$ (Figure 6E), confirming PIF1-

254 mediated suppression of MIR408 in planta.

255 To monitor the influence of PIF1 on miR408 and PLC transcript accumulation, we

256 performed RT-qPCR analysis of the pif1 and PIF1-OX lines. This analysis revealed that miR408

257 abundance significantly increased in pifl but decreased in PIF1-OX in both phy $\mathrm{A}_{\mathrm{OFF}}$ and phy $\mathrm{A}_{\mathrm{ON}}$

258 with reference to the wild type (Figure 6F). Conversely, PLC abundance significantly increased

259 in PIF1-OX but decreased in pifl in both phyA $\mathrm{OFF}_{\text {and phy }}$ an (Figure 6G). Taken together,

260 these results demonstrate that PIF1 binds to the MIR408 promoter and represses accumulation of

261 miR408 in the seed, which in turn post-transcriptionally silences $P L C$, thereby forming a PIF 1-

262 MIR408-PLC repression cascade.

264 PIF1, MIR408, and PLC Act in the Same Pathway to Regulate Germination

265 Consistent with previously reports (Oh et al., 2004), we found that pif1 seed completely 
germinated by $72 \mathrm{~h}$ in both phyA $\mathrm{OFF}_{\mathrm{F}}$ and phyA $_{\mathrm{ON}}$ (Figure 7A). This enhanced germination

267 phenotype was observed for the miR408-OX and plc seeds as well (Figure 2B and 5B).

268 Conversely, PIF 1-OX seed failed to germinate even by $120 \mathrm{~h}$ in phyA 269 that amiR408 and $\beta$-estradiol treated $i P L C-O X$ seeds exhibited similar phenotypes like PIF1-OX

270 (Figure $2 \mathrm{~F}$ and 5C). These results indicate that the molecularly delineated PIF 1-MIR408-PLC 271 repression cascade was in line with the germination phenotypes of the relevant mutants.

272 To further confirm that PIF1, MIR408, and PLC acts in the same genetic pathway, we 273 generated and analyzed three double mutants involving pif1 and PIF1-OX. We found that 274 germination rate of the pifl amiR408 seed was substantially reduced compared to that of pifl

275 (Figure 7A). By $120 \mathrm{~h}$ in phy $\mathrm{A}_{\mathrm{OFF}}$ and phy $\mathrm{A}_{\mathrm{ON}}$, the near complete germination of pifl was 276 lowered to about $40 \%$ and $80 \%$ by amiR408, respectively (Figure 7A). Regarding the PIF $1-O X$ miR408-OX double overexpression seed, over $20 \%$ germinated by $120 \mathrm{~h}$ in phy $\mathrm{A}_{\mathrm{ON}}$ (Figure $7 \mathrm{~B}$ ),

278 indicating that $m i R 408-O X$ was able to partially rescue the germination defect of PIF1-OX.

279 These results demonstrate that MIR408 is downstream of PIF1 in the same pathway. We also 280 tested the genetic relationship between PLC and PIF 1 by generating the PIF 1-OX plc line. We

281 found that the germination profile of this line was similar to that of the wild type with

282 substantially increased rates than PIF1-OX in both phy $\mathrm{A}_{\mathrm{OFF}}$ and phyA $\mathrm{ON}_{\mathrm{ON}}$ (Figure 7C), indicating 283 that $P L C$ is also downstream of PIF1. Thus, PIF1, MIR408, and PLC act sequentially in the 284 same pathway to regulate seed germination.

\section{PIF1, MIR408, and PLC Regulate Overlapping Cohorts of Genes}

287 As a master transcriptional regulator, PIF1 programs the seed germination related transcriptome 288 (Oh et al., 2009; Shi et al., 2013; Pfeiffer et al., 2014). To test whether that transcriptome is 289 regulated through the PIF1-MIR408-PLC pathway, we performed RNA-sequencing analysis of 290 the wild type, pif1, and miR408-OX seeds imbibed in darkness. The $i P L C-O X$ imbibed seed with 291 or without $\beta$-estradiol treatment was also analyzed. Consistent with previous reports (Oh et al., 292 2009; Shi et al., 2013), we identified 5,640 genes that were differentially expressed between pif1 293 and the wild type, which was defined as the PIF1-regulated set (Figure 8A). Genes differentially 294 expressed between $m i R 408-O X$ and the wild type were defined as the MIR408-regulated set, 295 which included 4,294 genes (Figure 8A). Differentially expressed genes in $i P L C-O X$ with and 296 without $\beta$-estradiol treatment were defined as the $P L C$-regulated set, which included 14,646 
genes (Figure 8A).

Venn diagram analysis revealed that there were 2,651 common genes between the PIF1regulated and the MIR408-regulated sets, 4,482 common between the PIF 1-regulated and the $P L C$-regulated sets, and 3,294 common between the MIR408-regulated and the PLC-regulated sets (Figure 8A). Based on Pearson correlation coefficient of fold changes against the controls, we found that these common genes exhibited high pairwise correlations between the compared genotypes (Figure 8B). Venn diagram analysis further revealed 2,290 genes that were differentially expressed in pif1, miR408-OX, and $i P L C-O X$ compared to the respective controls (Figure 8A). Clustering analysis showed that the vast majority of these genes was regulated in the same direction in pifl and miR408-OX but the opposite direction in $i P L C-O X$ (Figure 8C). Together these results indicate that the PIF1-MIR408-PLC pathway regulates large cohorts of common target genes in the seed.

Since $79.5 \%$ of the $P I F 1$-related genes, or 4,482 out of 5,640, was differentially regulated in $i P L C-O X$ (Figure 8A), which also exhibited the highest pairwise correlation among the genotypes $(\mathrm{R}=0.884$; Figure $8 \mathrm{~B})$, we selected the PIF1-PLC coregulated genes for further

312 analyses (Supplemental Dataset 1). Gene Ontology (GO) analysis revealed that the PIF1-PLC

313 coregulated genes were preferentially associated with terms in two categories: seed development

314 and germination, and hormone metabolism and signaling (Supplemental Figure 3). For example,

315 the GO term "seed germination" was associated with 218 genes in Arabidopsis (Supplemental

316 Dataset 2). These genes could be divided into three groups based on their expression pattern in

317 pifl (Figure 8D). Genes in group I (40 out of 218, or 18.3\%) and III (41 out of 218, or 18.8\%)

318 were substantially down-regulated and up-regulated in pifl, respectively. They were reversely 319 modulated in $i P L C-O X$ (Figure 8D).

320 Further inspection revealed that many individual genes that have been genetically or

321 functionally implicated in germination related processes were included in group I and III

322 (Supplemental Figure 4). For example, SOM and RVE2 from group I were reported to inhibit 323 light dependent germination (Kim et al., 2008; Jiang et al., 2016). On the contrary, JMJ22 and

324 MAN7 from group III were reported to promote seed germination (Iglesias-Fernández et al., 2011;

325 Cho et al., 2012). These two types of genes were regulated in an opposite pattern in pifl and $326 i P L C-O X$ (Figure 8E). Collectively, these results indicate that PLC is a key node downstream of

327 PIF1 to mediate the transcriptomic changes underlying light dependent germination. 


\section{The PIF1-MIR408-PLC Pathway Regulates GA and ABA Biosynthesis}

As rapid removal of PIF1 is critical for ultimately establishing the high-GA-low-ABA state, we examined whether PLC impacts the GA-ABA balance. To this end, we first inspected gene expression profile along the GA metabolic pathway. This analysis revealed that the PIF1MIR408-PLC pathway regulates in particular GA3ox1 and GA2ox2, which encode the GA3oxidase that catalyzes the terminal GA biosynthetic step and the GA2-oxidase that catabolizes bioactive GAs, respectively (Figure 9A) (Yamaguchi, 2008). This finding was corroborated by RT-qPCR analysis showing that GA3oxl was up-regulated in pif1, miR408-OX, and plc, but down-regulated in PIF1-OX and amiR408 seeds compared to the wild type in both phy $\mathrm{A}_{\mathrm{OFF}}$ and phyA $_{\mathrm{ON}}$ (Figure 9B). In contrast, GA2ox2 was up-regulated in PIF1-OX and amiR408 but downregulated in pif1, miR408-OX, and plc seeds relative to the wild type (Figure 9B). These results indicate that the PIF1-MIR408-PLC pathway targets the GA3ox and GA2ox steps in GA biosynthesis to regulate the level of bioactive GA.

Next, we directly quantified the amount of bioactive GA in the seed using the ultrahigh-

343 performance liquid chromatography-triple quadrupole mass spectrometry (UPLC-MS/MS) method (Fu et al. 2012; Ma et al., 2015). Compared to the wild type, level of GA4, the major bioactive GA in Arabidopsis seed (Oh et al., 2006), was significantly elevated in pif1, miR408$O X$, and plc seeds (Figure 9C). In PIF 1-OX and amiR408, GA 4 level was significantly reduced compared to the wild type (Figure 9C). These results indicate that the PIF1-MIR408-PLC pathway is capable of modulating the level of bioactive GA in the seed.

We further performed pharmacological analyses using bioactive $\mathrm{GA}_{3}$ and paclobutrazol, an inhibitor of GA biosynthesis. We found that $\mathrm{GA}_{3}$ application promoted all seeds, including

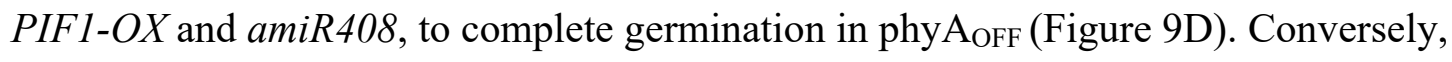
paclobutrazol blocked germination of all seeds, including pifl, miR408-OX, and plc, in phyA

353 (Figure 9D). Taken together, the gene expression, hormone quantification, and pharmacological 354 results demonstrate that the PIF1-MIR408-PLC cascade regulates germination by modulating the 355 level of bioactive GA in the seed.

356 Transcriptomic profiling also revealed that $A B A 1, N C E D 6$ and NCED 9 were among the 357 most substantially influenced ABA biosynthetic genes, which were downregulated in pifl and 358 miR408-OX but upregulated in $\beta$-estradiol treated $i P L C-O X$ seeds compared to the controls 
359 (Figure 10A). RT-qPCR analysis confirmed that $A B A 1, N C E D 6$ and NCED9 were positively

360 regulated by $P I F 1$ and $P L C$ but negatively regulated by miR408 in both phy $A_{\mathrm{OFF}}$ and phy $\mathrm{A}_{\mathrm{ON}}$

361 (Figure 10B). Chemical quantification showed that endogenous ABA level in pif1, miR408-OX,

362 and plc seeds was significantly reduced compared to that in the wild type (Figure 10C).

363 Furthermore, we found that pharmacological treatment with ABA blocked germination of all

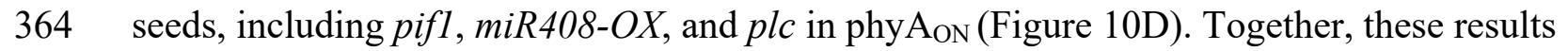

365 indicate that the PIF1-MIR408-PLC pathway regulates germination through reciprocally

366 modulating the biosynthesis of GA and ABA.

\section{PLC Is Conserved in Seed Plants}

369 Finally, to provide phylogenetic evidence supporting PLC as a key node in germination, we examined PLC conservation in seed plants. Searching for putative PLC orthologs from sequenced land plant genomes identified 276 PLC sequences from 52 seed plants but not nonseed plants (Figure 11; Supplemental Figure 5). Comparison of PLC sequences and domain organization to the most homologous blue copper proteins in three representative non-seed plants

374 revealed two salient features of PLC. First, all PLCs were found to contain only a signal peptide

375 at the N-terminus and a type-I copper binding motif at the C-terminus (Figure 11; Supplemental

376 Figure 5). These two domains exhibited an extremely compact organization. For example, the

377 Arabidopsis PLC possessed 129 amino acid residues of which 33 (25.6\%) were devoted to the

378 signal peptide and $95(73.6 \%)$ to the copper binding motif (Figure 11). Second, a miR408

379 recognition site was found near the 5' end of the coding region of all examined PLC transcripts

380 (Figure 11), which has been experimentally validated in several plant species (Abdel-Ghany and

381 Pilon, 2008; Zhou et al., 2010; Feng et al., 2013; Zhang and Li, 2013). Moreover, length and

382 domain organization suggest that PLC in Ginkgo may resemble the prototype of this protein,

383 which was further evolved in angiosperm by trimming the C-terminus to a bare-bones copper

384 binding motif (Figure 11). Taken together, our results indicate that PLC has specifically evolved

385 in seed plants as a miR408 targeted, storage vacuole associated compact cuproprotein, which

386 balances GA and ABA levels for controlling germination (Figure 12). 
bioRxiv preprint doi: https://doi.org/10.1101/2020.07.20.212340; this version posted July 21, 2020. The copyright holder for this preprint (which was not certified by peer review) is the author/funder. All rights reserved. No reuse allowed without permission.

387 


\section{DISCUSSION}

389 The seeds are equipped with elaborate molecular mechanisms to monitor and transduce the light

390 signals for proper germination, which is vital for survival of seed plants (Oh et al., 2004; Finch-

391 Savage and Leubner-Metzger, 2006; Seo et al., 2009; Shi et al., 2015). Decades of research has

392 shown that a decisive event downstream of light signaling is establishment of the high-GA-low-

393 ABA hormonal state (Nambara and Marion-Poll, 2005; Oh et al., 2006; 2007; Yamaguchi, 2008;

394 Seo et al., 2009; Shu et al., 2016). Elucidating how the light signals are converted into the

395 hormonal profiles is critical to our understanding of seed biology, which bears immediate

396 relevance to agriculture and human nutrition.

\section{A Long Repression Cascade Regulating Light Dependent Germination}

399 In this study, we used far-red light triggered, phyA dependent germination as the experiment

400 model (Oh et al., 2004; 2006; Cho et al., 2012). Our comprehensive molecular and genetic

401 analyses (Figure 1 to 7), incorporated to established light signaling framework (Castillon et al.,

402 2007; Leivar and Quail, 2010), delineated the phyA-PIF1-miR408-PLC repression cascade as a

403 key regulatory mechanism in germination (Figure 12). Through transcriptome profiling and

404 quantification of endogenous GA and ABA levels, we found that this signal relay chain regulates

405 the conversion of light signals into hormonal profiles (Figure 8 and 10). In darkness, absence of

406 active phyA leads to PIF1 accumulation (Shen et al., 2008), which suppresses transcription of

407 MIR408 (Figure 6). Low level of miR408 in turn allows PLC to accumulate in the storage

408 vacuole (Figure 1 and 4), which correlates with the low-GA-high-ABA state (Figure 9 and 10).

409 Upon far-red irradiation, phyA is activated to rapidly destabilize PIF1 (Oh et al., 2007; Shen et

410 al., 2008), releasing MIR408 from transcriptional inhibition (Figure 6). Accumulation of miR408

411 then leads to PLC silencing (Figure 1 and 4), which correlates with the high-GA-low-ABA state

412 (Figure 9 and 10). This chain of events forms a multistep repressor cascade typical for

413 developmental transcription networks, which generates robust temporal delay (Rosenfeld and

414 Alon, 2003; Shoval and Alon, 2010). The phyA-PIF1-miR408-PLC repression cascade therefore

415 may help to specify the time from light perception to PLC turnover.

416 Based on phylogenetic findings, the phyA-PIF1-miR408-PLC cascade appears to have

417 formed by sequentially adding downstream components during evolution of the seed plants. The

418 phytochrome signaling pathway and the PIF family were shown to originate in the ancestors of 
419 charophytes (Han et al., 2019). Inhibition of PIFs by phytochromes to regulate light responses

420 was conserved at least in vascular plants and the liverworts (Lee and Choi, 2017; Han et al.,

421 2019). On the other hand, miR408 is deeply conserved in land plants including moss (Pan et al.,

422 2018; Guo et al., 2020) while PLC acquired the miR408 recognition site after the emergence of

423 seed plants (Figure 11). These observations suggest that the repression cascade has specifically

424 evolved in seed plants, taking advantage of extant regulatory modules, for controlling seed

425 germination.

426 HY5 and PIFs are known to function antagonistically to adjust the expression of common

427 target genes related to biological processes such as seedling establishment, photosynthetic

428 pigment synthesis, production of reactive oxygen species, and phosphate starvation response

429 (Chen et al., 2013; Toledo-Ortiz et al., 2014; Sakuraba et al., 2018; Shi et al., 2018). We reported

430 previously that HY5 binds to the G-box in the MIR408 promoter and promotes miR408

431 accumulation in young seedlings in a light intensity dependent manner (Zhang et al., 2014). The

432 discovery of PIF1 represses MIR408 transcription via binding to the G-box (Figure 6) indicates

433 that PIF1 and HY5 reciprocally and sequentially control miR408 accumulation before and after

434 germination (Figure 12). This circuit may form a switch for optimizing germination and the

435 ensued post-germinative growth in light (Figure 12).

\section{PLC Links Copper Mobilization to Hormone Metabolism}

438 PLC belongs to the phytocyanin family of small blue copper proteins, which are ancient copper-

439 containing redox proteins widely distributed in microorganisms and plants (Rydén and Hunt

440 1993; Guss et al., 1998; De Rienzo et al. 2000; Giri et al. 2004). Different from other

441 phytocyanins, PLCs are extremely compact with the 120-130 amino acid residues devoting to

442 three conserved motifs. Besides the characteristic type-I copper binding motif on the C-terminus,

443 the signal peptide and the miR408 recognition site superimpose on the N-terminus (Figure 11;

444 Supplemental Figure 5). Our results provided two important clues to PLC function. First, through

445 quantification of endogenous hormones, genetic analysis, gene expression profiling, and

446 pharmacological analyses (Figure 8 to 10), we demonstrated that PLC acts as a switch

447 downstream of PIF1 and is both necessary and sufficient to reciprocally modulate GA and ABA

448 levels in the seed. Previously, high expression of $P L C$ in the transmitting tract of the pistil was

449 noticed (Dong et al. 2005). Over-expression of PLC was found to disrupt pollen tube guidance 
450 into the style and to reduce seed set (Dong et al. 2005). The latter was corroborated by

451 observations that over-expression of miR408 resulted in larger seed size and higher grain yield

452 (Pan et al., 2018). Thus, besides seed germination, PLC may participate in other aspects of seed

453 biology and reproductive development. It will be interesting to test if rebalancing endogenous

454 hormone levels is the unifying function of PLC in these processes.

455 Second, through RNA-sequencing, we found that PLC is both necessary and sufficient to

456 regulate an overwhelming portion of the PIF1-dependent transcriptome underlying germination

457 (Figure 8; Supplemental Figure 3; Supplemental Dataset 1). This finding suggest that PLC

458 turnover is associated with major changes to cellular state. Because PLC is a vacuole located

459 cuproprotein with a bare-bones type-I copper motif and highly expressed in mature seed (Figure

4601 and 11), we contemplated that PLC is a key carrier for copper mobilization. In the seed,

461 mineral nutrients are sequestered in the storage vacuoles (Lanquar et al., 2005; Kim et al., 2006;

462 Roschzttardtz et al., 2009; Eroglu et al., 2017). Right after imbibition, there likely is no new

463 assimilation of transition metals before massive translation and protein synthesis is taking place

464 (Née et al., 2017; Paszkiewicz et al., 2017). Thus, mineral elements need to be mobilized from

465 vacuolar stores and transported to the cytoplasm and other organelles for reconstituting

466 biochemical activity. Disrupting these processes has been shown to lead to severe germination

467 defects (Lanquar et al., 2005; Kim et al., 2006). Previously we have shown that miR408

468 promotes copper allocation to the plastid and enhances photosynthesis via elevating plastocyanin

469 abundance (Zhang et al., 2014; Pan et al., 2018). Taken together, we speculate that the miR408-

$470 \quad P L C$ module controls copper redistribution between the vacuole and the plastid.

\section{How Does PLC Regulate GA and ABA Biosynthesis?}

473 PLC turnover as a means for copper mobilization and delivery to the plastid is consistent with

474 previous studies on the effects of copper on plastid physiology and biochemistry. Plastid is

475 known to be the major cellular copper sink in plants (Burkhead et al., 2009), whereby the

476 transition metal acts as cofactor for plastocyanin in the thylakoid lumen, which is indispensable

477 as an electron carrier in the Z-scheme of photosynthesis (Molina-Heredia et al., 2003; Weigel et

478 al., 2003), and for the copper- and zinc-containing superoxide dismutase in the stroma, which

479 participates in neutralizing reactive oxygen species to maintain proper redox state in the plastid

480 (Gupta et al., 1993). Copper allocation to the plastid was shown to be critical for plastocyanin 
481 abundance and activity (Weigel et al., 2003; Zhang et al., 2014; Pan et al., 2018). Copper level 482 was also reported to impact the number of chloroplasts per cell, thylakoid stacking, and grana 483 size (Bernala et al., 2006). It is intriguing to note that GA biosynthesis initiates in the plastid 484 (Sun and Kamiya 1997; Yamaguchi, 2008). Thus, regulated PLC degradation may promote 485 copper translocation or allocation to the plastid. Copper-propelled plastid development may in turn provide the structural and biochemical niche for initiating GA biosynthesis in the seed.

Alternatively, the effect of PLC turnover on hormone rebalancing may be explained by a direct impact on ABA synthesis taking place in the cytosol. In Arabidopsis, AAO3 encodes an aldehyde oxidase that catalyzes the last step of ABA biosynthesis, the conversion of abscisic aldehyde to ABA (Seo et al., 2000). AAO3 is a cytosolic molybdoenzyme that requires the molybdenum cofactor for catalytic activity (Seo et al., 2000). Structural and biochemical analyses have shown that the final step of molybdenum cofactor biosynthesis is dependent on a

493 copper-dithiolate complex, which protects the reactive dithiolate before molybdenum insertion

494 (Kuper et al., 2004). It could be speculated that PLC is one of the copper donors, through 495 unidentified cytoplasmic chaperones, passes on copper to the dithiolate group for synthesizing 496 the molybdenum cofactor (Peñarrubia et al., 2015). Induction of PLC expression during late seed 497 development (Figure 1) is consistent with this scenario whereby elevated PLC helps to maintain 498 AAO3 activity and hence ABA accumulation during seed maturation. Upon light irradiation, 499 rapid PLC turnover would deplete copper supply for AAO3 and impede ABA synthesis, which is 500 consistent with the fourfold decline of ABA content in the plc seed over the wild type (Figure 501 10C). The finding in rice that exogenous copper increases ABA accumulation and inhibits 502 germination (Ye et al., 2014) provides another line of evidence for this model. Further studying 503 PLC-related copper homeostasis could shed more light on hormone synthesis and balancing 504 during seed development and germination. 
bioRxiv preprint doi: https://doi.org/10.1101/2020.07.20.212340; this version posted July 21, 2020. The copyright holder for this preprint (which was not certified by peer review) is the author/funder. All rights reserved. No reuse allowed without permission.

505 


\section{METHODS}

\section{Plant Materials and Growth Conditions}

The wild type Arabidopsis thaliana used in this study was Col-0. The pif1, PIF1-OX, pMIR408:GUS, miR408-OX, and amiR408 plants were as previously described (Oh et al., 2004;

510 Zhang and Li, 2013; Zhang et al., 2014). To delete PLC, a CRISPR/Cas9 system employing the 511 modified pCAMBIA1300 vector was used (Mao et al., 2013) in which the 35S and the AtU6-26

512 promoter respectively drive $\operatorname{Cas} 9$ and a pair of $\operatorname{sgRNAs}$ that were designed to target both ends of

513 the $P L C$ coding region. The resulting construct was used to transform the wild type and $\mathrm{T}_{1}$ plants

514 were individually genotyped by PCR and sequencing to identify deletion events. Approximately

515100 individual $\mathrm{T}_{2}$ plants were genotyped to identify Cas 9 -free homozygous $p l c-2$ lines. The

$516 i P L C-O X$ transgenic plants were obtained by cloning the $P L C$ coding sequence into the pER8

517 vector (Zuo et al., 2000) and transforming wild type plants. Homozygotes were selected for

518 Hygromycin resistance in the $\mathrm{T}_{2}$ population. The $p P L C: P L C$-GFP transgenic plants were

519 obtained by cloning the PLC coding sequence into the modified pJim19-GFP vector and

520 substituting the $P L C$ promoter for the $35 S$ promoter. Following transformation of the wild type

521 plants, homozygotes were selected by Kanamycin resistance in the $\mathrm{T}_{2}$ population. Sequences of

522 the relevant primers are listed in Supplemental Table 1. The pMIR408:GUS/pif1, PIF 1-OX

523 miR408-OX, pif1 amiR408, PIF1-OX plc, lac12 lac13, and plc lac12 lac13 lines were generated

524 by crossing and selection for homozygotes at the $\mathrm{F}_{2}$ generation.

525 Adult Arabidopsis plants were grown in soil at $22^{\circ} \mathrm{C}, \sim 60 \%$ relative humidity, and under

526 long day (16 h light/8 h dark) condition in a growth chamber. For each experiment, the seeds

527 were harvested at approximately the same time. After harvesting, the seeds were dried at room

528 temperature for six to eight weeks prior to germination and other experiments.

Germination Assays

531 The far-red light induced germination assay was performed as described with minor

532 modifications (Oh et al., 2004). Briefly, a triplicate set of 50-75 seeds for each sample was

533 surface sterilized with liquid bleach and plated on half-strength MS aqueous agar medium $(0.6 \%$

534 agar, 1\% sucrose, $\mathrm{pH}$ 5.7). One hour after the start of sterilization, the plated seeds were

535 irradiated with $3.2 \mu \mathrm{M} \mathrm{m}^{-2} \mathrm{~s}^{-1}$ far-red light for $5 \mathrm{~min}$ and then incubated in the dark for $48 \mathrm{~h}$. For 536 phy $A_{\mathrm{OFF}}$, the imbibed seeds were continuously placed in darkness for up to $120 \mathrm{~h}$. For phy $\mathrm{A}_{\mathrm{ON}}$, 
537 the seeds were treated with a second far-red irradiation for $4 \mathrm{~h}$ and then in darkness for up to 120

538 h. For pharmacological analysis, $100 \mu \mathrm{M}$ paclobutrazol, $10 \mu \mathrm{M} \mathrm{GA}_{3}$, or $5 \mu \mathrm{M}$ ABA (Sigma-

539 Aldrich) was supplemented to the medium. Germination was determined by examining radicle

540 formation at the indicated time points.

542 Analysis of Seedling Greening

543 The seedlings were grown in dark for four days and then transferred to continuous white light

$544\left(100 \mu \mathrm{M} \mathrm{m}^{-2} \mathrm{~s}^{-1}\right)$ for $24 \mathrm{~h}$. The greening rate was determined and calculated as the ratio of green

545 seedlings over the total germinated seedlings as previously described (Zhong et al., 2009).

546 Pigments were extracted from etiolated seedlings in the dark at room temperature using $90 \%$

547 acetone containing $0.1 \% \mathrm{NH}_{3}$ as previously described (Zhong et al., 2014). Supernatants

548 containing the pigments were subject to fluorescence spectral analysis using an Infinite M200

549 microplate reader (Tecan). The excitation wavelength was $443 \mathrm{~nm}$ and the emission spectra were

550 recorded from 610 to $740 \mathrm{~nm}$ with $1 \mathrm{~nm}$ bandwidth. All measurements were performed on at

551 least three independent biological samples and one representative set of results was shown.

\section{Transcript Quantification}

554 Total RNA from imbibed seeds was isolated using the Quick RNA Isolation Kit (Huayueyang).

555 For each experiment, mRNA and miRNA from three independent biological samples were

556 reverse transcribed into cDNA using the PrimeScript II $1^{\text {st }}$ Strand cDNA Synthesis Kit (TaKaRa)

557 and the miRcute Plus miRNA First-Stand cDNA Synthesis Kit (Tiangen), respectively. qPCR

558 was performed using SYBR Green Mix (TaKaRa) on the 7500 Fast Real-Time PCR System

559 (Applied Biosystems). Actin7 and 5S RNA were used for mRNA and miRNA normalization,

560 respectively. Sequences of the primers are listed in Supplemental Table 1.

\section{RNA-sequencing Analysis}

563 Seeds of the wild type, miR408-OX and pif1 were grown on half-strength MS medium. The

$564 i P L C$ - $O X$ seed grown on medium supplemented with or without $5 \mu \mathrm{M} \beta$-estradiol (Sigma-

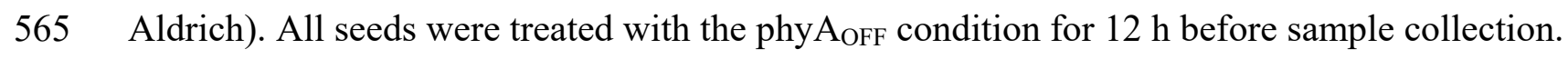

566 Total RNA was isolated using the Quick RNA Isolation Kit (Huayueyang). Library preparation

567 and RNA-sequencing were performed on the Illumina HiSeq 2000 platform. For each genotype, 
three paired-end libraries from independent biological samples were prepared. At least 16 million raw paired-end reads were generated from each library. Quality control was conducted using fastQC. Cutadapt and a custom Perl script were used to trim adaptors with the parameter Q30 and the first nine bases following the adaptors with low fastQC score. After trimming, only

572 reads longer than 100 bases were retained and the R1 and R2 files were paired simultaneously.

573 The clean reads were mapped to the TAIR10 Arabidopsis genome build using STAR with an

574 average mapping rate of $\sim 90 \%$ and unique mapping rate above $80 \%$. Transcript quantification

575 results generated by Stringtie were processed by Cuffdiff to identify differentially expressed genes. Clustering and correlation analyses were performed and visualized using R scripts. GO analysis was carried out using AgriGO (http://bioinfo.cau.edu.cn/agriGO/).

\section{ChIP-qPCR}

580 ChIP was carried out on four-day-old dark-grown PIF1-OX and wild type seedlings on MS medium using an anti-MYC polyclonal antibody (Sigma-Aldrich) as described (Pfeiffer et al., 2014). After ChIP, equal amount of input DNA was subjected to qPCR analysis of the target DNA fragment. Fold of enrichment was calculated between PIF1-OX and the wild type input.

\section{Immunoblotting}

586 The $p P L C: P L C$-GFP line was used for protein analysis. Dry seed was incubated in water at 587 room temperature for $0.5 \mathrm{~h}$ or at $4^{\circ} \mathrm{C}$ for $24 \mathrm{~h}$ before sample collection. Total protein was 588 isolated with an extraction buffer containing $50 \mathrm{mM}$ Tris $\cdot \mathrm{HCl}, \mathrm{pH} 7.5,6 \mathrm{mM} \mathrm{NaCl}, 1 \mathrm{mM}$

$589 \mathrm{MgCl}_{2}, 1 \mathrm{mM}$ PMSF, and $1 \times$ protease inhibitor mixture (Roche). Immunoblotting was performed 590 with an anti-GFP antibody (Abcam). An anti-RPT5 antibody (Abcam) was used as loading 591 control.

\section{Histochemical Staining for GUS Activity}

594 After stripping away the seed coat in green safe light, pMIR408:GUS and pMIR408:GUS/pif1

595 seeds grown for $12 \mathrm{~h}$ in phy $\mathrm{A}_{\mathrm{OFF}}$ and phy $\mathrm{A}_{\mathrm{ON}}$ were incubated in a standard GUS staining

596 solution for $3 \mathrm{~h}$ at $37^{\circ} \mathrm{C}$. Following removal of the staining solution, seeds were washed with

597 several changes of $75 \%$ ethanol until pigments were no longer visible. Images of the GUS

598 staining pattern were taken with a digital camera. 


\section{Live-cell Imaging}

601 The $p P L C: P L C-G F P$ seed was treated with phy $\mathrm{A}_{\mathrm{OFF}}$ and phy $\mathrm{A}_{\mathrm{ON}}$ for the indicated duration.

602 Cotyledons were dissected away from the testa and endosperm by the application of gentle

603 pressure to seed held between a microscope slide and a cover slip. Fluorescence images were

604 obtained using a Nikon A1R si+ laser scanning confocal microscope equipped with an APO

$60540 \times 1.25 \mathrm{NA}$ water immersion objective. Excitation and emission wavelengths were 488/500 to

$606550 \mathrm{~nm}$ for GFP and 405/425 to $475 \mathrm{~nm}$ for vacuole autofluorescence. Autofluorescence spectra

607 were obtained using a 32-PMT spectral detector. Spectral unmixing and image analysis were

608 performed using the NIS Elements AR software (Nikon Instruments).

609 Transient expression in onion epidermal cells was performed as previously described

610 (Wang and Frame, 2009). The gold particles were coated with plasmid DNA containing the

611 expression cassette for PLC-GFP or COPT5-mCherry. The Biolistic PDS-1000/He Particle

612 Delivery System (Bio-Rad) was used for bombarded with the following settings: 1,100 psi

613 rupture disc, 25-26-inch $\mathrm{Hg}$ vacuum, and target distance of $10 \mathrm{~cm}$. After bombardment, the

614 explants were kept in dark at $25^{\circ} \mathrm{C}$ for $16-18 \mathrm{~h}$ and observed with the Nikon A1R si+ microscope.

615 Excitation and emission wavelengths were 488/500 to $550 \mathrm{~nm}$ for GFP and 561/570 to $620 \mathrm{~nm}$

616 for mCherry.

\section{Quantification of Endogenous GA and ABA}

619 For each genotype, $500 \mathrm{mg}$ of seed grown in phy $\mathrm{A}_{\mathrm{OFF}}$ for $24 \mathrm{~h}$ was collected and ground into

620 fine powder in liquid nitrogen. Endogenous ABA was purified and measured as previously

621 described (Fu et al. 2012) with minor modifications to the detection procedure. Briefly, UPLC-

$622 \mathrm{MS} / \mathrm{MS}$ analysis was performed on a UPLC system (Waters) coupled to the 5500 Qtrap system

623 (AB SCIEX). Chromatography separation was achieved with a BEH C18 column (Waters) with

624 mobile phase $0.05 \%$ HAc (A) and $0.05 \%$ HAc in ACN (B). The gradient was set initially with 20\%

$625 \mathrm{~B}$ and increased to $70 \% \mathrm{~B}$ within 6 min. ABA was detected in the MRM mode with transition

$626263 / 153$ and the isotope dilution method was used for quantification.

627 Quantitative GA measurement was performed as previously described using UPLC-

628 MS/MS (Ma et al., 2015). Chromatography separation was achieved with a BEH C18 column

629 (Waters) with mobile phase 0.05\% HAc (A) and 0.05\% HAc in ACN (B). Gradient was set as 
630 the following: $0-17 \mathrm{~min}, 3 \% \mathrm{~B}$ to $65 \% \mathrm{~B}$; $17-18.5 \mathrm{~min}, 65 \% \mathrm{~B}$ to $90 \% \mathrm{~B} ; 18.5-19.5 \mathrm{~min}, 90 \% \mathrm{~B}$;

$631 \quad 19.5-21 \mathrm{~min}, 90 \% \mathrm{~B}$ to $3 \% \mathrm{~B}$; and $21-22.5 \mathrm{~min}, 3 \% \mathrm{~B}$. $\mathrm{GA}_{4}$ was detected in the negative MRM

632 mode and quantified with a MRM transition. The source parameters were set as IS voltage -4500

$633 \mathrm{~V}, \mathrm{TEM} 600^{\circ} \mathrm{C}, \mathrm{GS} 145, \mathrm{GS} 255$, and curtain gas 28.

634

635 Transient Expression in Tobacco Protoplasts

636 The promoter sequence of MIR408 was cloned from Arabidopsis genomic DNA, inserted into

637 the pGreen II 0800-LUC vector (Hellens et al., 2005), and used as the reporter. The PIF1 coding

638 sequence was cloned from Arabidopsis cDNA, inserted into pGreen II 62-SK (Hellens et al.,

639 2005), and used as the effector. Tobacco protoplasts were freshly prepared as described

640 previously (Yoo et al., 2007). The effector or the empty vector was combined with the report

641 construct and used to transiently transform the protoplasts using the Dual-Luciferase Reporter

642 System (Promega), following the manufacturer's instruction. Transfected protoplasts were

643 incubated under low light for $16 \mathrm{~h}$. The chemiluminescence was determined using a LB942

644 Multimode Reader (Berthold Technologies).

645

646 Domain and Phylogenetic Analyses of PLC

647 PLC in Arabidopsis was used as query to perform a BLASTP search against all proteins in 52

648 plant species covering all main clades of land plants. A total of 276 PLC sequences were

649 identified based on two criteria: E-value $\leq \mathrm{e}^{-10}$ and a "plantacyanin" annotation term assigned by

650 InterProScan. N-terminal signal peptide was predicted using SignalP (Almagro Armenteros et al.,

651 2019). Binding site for miR408 was predicted using psRNATarget (Dai et al., 2018). To show

652 the evolutionary trajectory, PLCs from 14 representative plant species and the most homologous

653 genes encoding small blue copper proteins from Physcomitrella patens, Salvinia cucullate, and

654 Azolla filiculoides were selected and mapped to a species tree obtained from TimeTree

655 (http://www.timetree.org/). 


\section{Supplemental Data}

657 Supplemental Figure 1. Generation and Characterization of PLC-related Mutants.

658 Supplemental Figure 2. The miR408-PLC Module Specifically Regulates Germination.

659 Supplemental Figure 3. Enriched GO Terms Associated with PIF1 and PLC Coregulated Genes.

660 Supplemental Figure 4. Exemplar Genes Regulated by the PIF1-PLC Pathway.

661 Supplemental Figure 5. PLC Has a Compact Domain Organization.

662 Supplemental Table 1. Oligonucleotide Sequences of the Primers Used in This Study.

663 Supplemental Dataset 1. List of PIF1 and PLC Coregulated Genes.

664 Supplemental Dataset 2. Expression Profile of 218 Germination Related Genes. 


\section{Accession Number}

666 Sequence data from this article can be found in the Arabidopsis Genome Initiative or

667 GenBank/EMBL databases under the following accession numbers: MIR408 (At2g47015), PIF1

668 (AT2g20180), HY5 (At5g11206), PLC (At2g02850), LAC3 (At2g30210), LAC12 (At5g05390),

669 LAC13 (At5g07130), GA2ox8 (At4g21200), GA3ox1 (At1g15550), GA2ox2 (At1g30040), ABA1

670 (At5g67030), NCED6 (At3g24220), NCED9 (At1g78390), SOM (At1g03790), RVE2

671 (At5g37260), JMJ22 (At5g06550) and MAN7 (At5g66460). T-DNA insertion mutants used are

672 pif1 (SALK_072677), plc-1 (SALK_091945), lac3 (SALK_031901C), lac12 (SALK_087122),

673 and lac13 (SALK_023935). RNA sequencing data can be found at the National Center for

674 Biotechnology Information Sequence Read Archive under accession number PRJNA633227.

675

676 Author Contributions

677 L.L. designed and supervised the research. A.J., J.P., Y.Z., D.Z., and C.H. performed the 678 experiments. Z.G. (Guo), Z.G. (Gao), and S.Z. analyzed the data. P.X. and J.C quantified

679 hormone levels. A.J. and L.L. wrote the paper.

680

\section{Acknowledgements}

682 We thank Drs. Xiangdong Fu, Xing-Wang Deng, and Hongwei Guo for providing some of the

683 plasmids and seeds used in this study. This work was supported by grants from the National Key

684 Research and Development Program of China (2017YFA0503800) and the National Natural

685 Science Foundation of China (31621001). 
bioRxiv preprint doi: https://doi.org/10.1101/2020.07.20.212340; this version posted July 21, 2020. The copyright holder for this preprint (which was not certified by peer review) is the author/funder. All rights reserved. No reuse allowed without permission.

686 
A
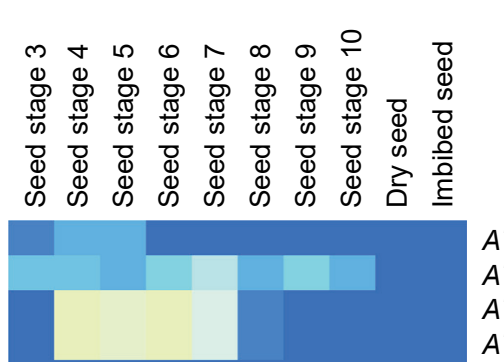

AT5G53870

AT4G27520

AT4G28365

AT4G32490

AT1G48940

AT1G79800

AT1G64640

AT3G20570

AT5G57920

AT2G23990

AT4G30590

AT5G25090

AT2G25060

AT4G31840

AT3G01070

AT5G15350

AT1G08500

AT4G12880

AT1G17800

PLC

AT5G20230

AT2G44790

AT3G60280

AT3G60270

AT3G27200

AT1G72230

AT1G22480

AT2G32300

AT5G26330

AT2G31050

AT2G26720

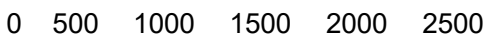

Expression signal

F

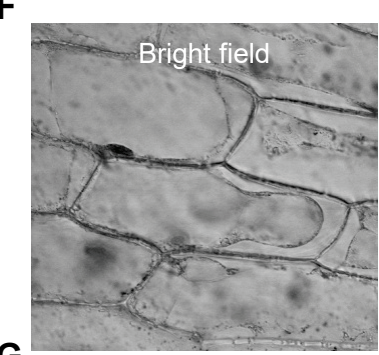

G

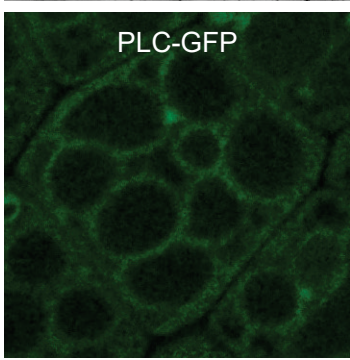

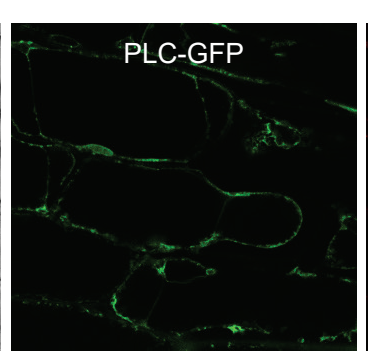

GFP merged with autofluorescence

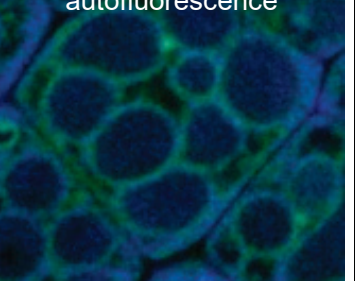

B
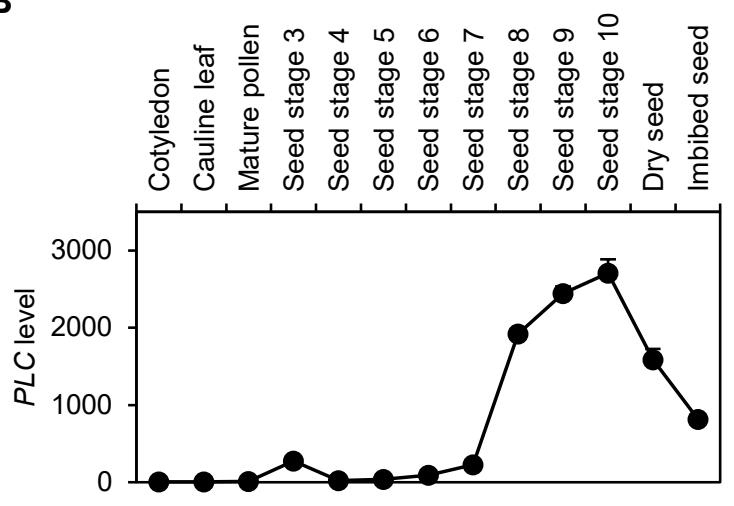

C

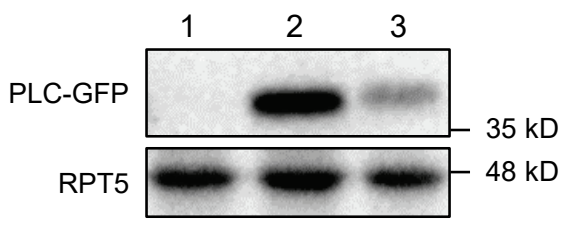

D

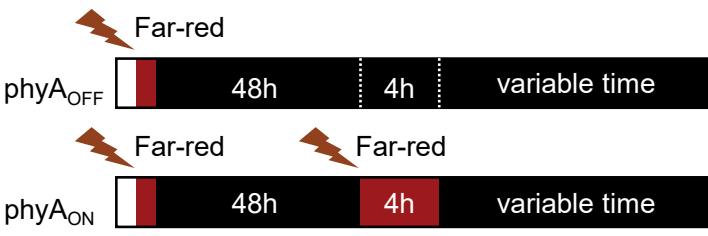

\section{E}
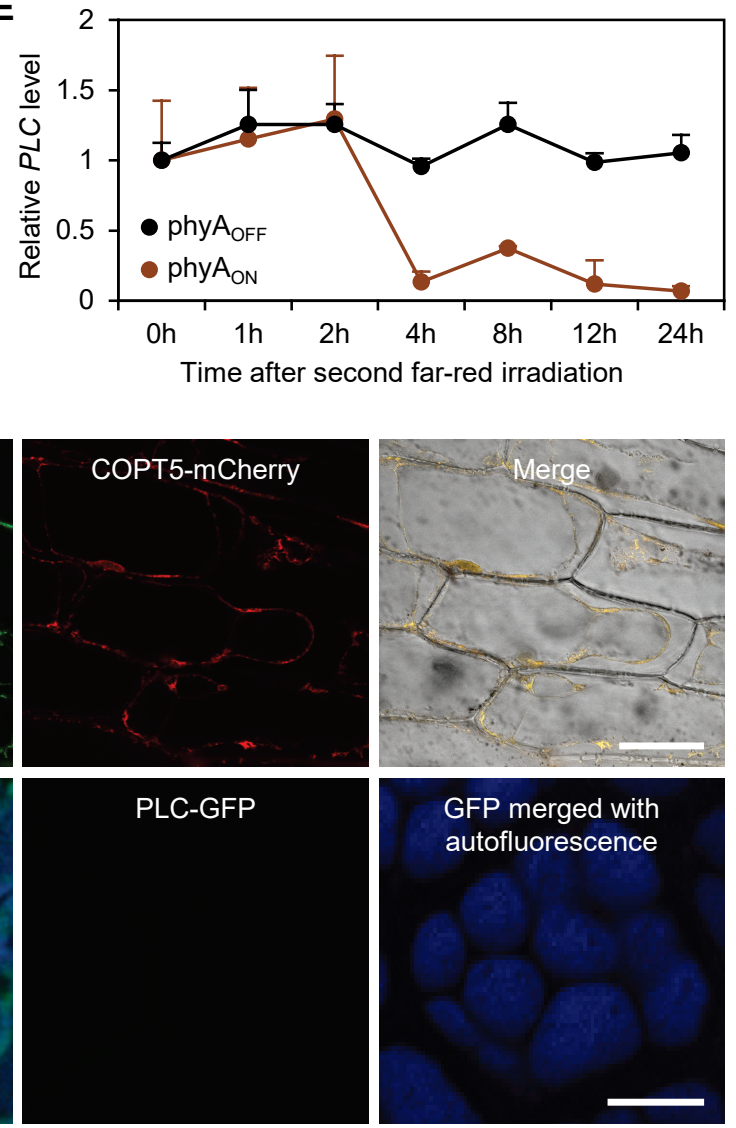

Figure 1. PLC Is Induced during Seed Development and Rapidly Silenced during Germination. 
Figure 1. PLC Is Induced during Seed Development and Rapidly Silenced during Germination.

(A) Global expression profile of 31 phytocyanin genes in the seed based on data in the Arabidopsis eFP Browser. (B) Comparison of PLC expression pattern in the seed and other organs, using data from the eFP Browser. (C) Detection of PLC-GFP in pPLC:PLC-GFP plants using an anti-GFP antibody. 1 , seedling; 2 , dry seed; 3 , vernalized seed. Size markers are indicated on the right. RPT5 was used as the loading control. (D) Diagram illustrating the phyA $\mathrm{OFF}_{\text {and }}$ phyA $\mathrm{ONN}_{\mathrm{N}}$ regimes in which imbibed seeds were sequentially treated with the indicated light conditions. (E) Relative PLC transcript level during the time course of phyA $\mathrm{OFF}_{\text {and }}$ any $\mathrm{A}_{\mathrm{ON}}$ determined by RT-qPCR analysis. Data are mean \pm SD $(n=3)$. (F) Subcellular localization of PLC. PLC-GFP and COPT5-mCherry were transiently expressed in the same onion epidermal cells and examined by confocal fluorescence microscopy. (G) Co-localization of GFP fluorescence with vacuole autofluorescence in

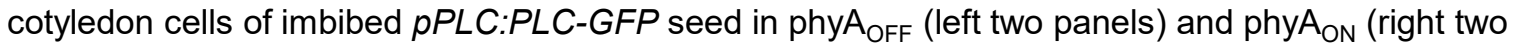
panels). Bars, $10 \mu \mathrm{m}$. 
A
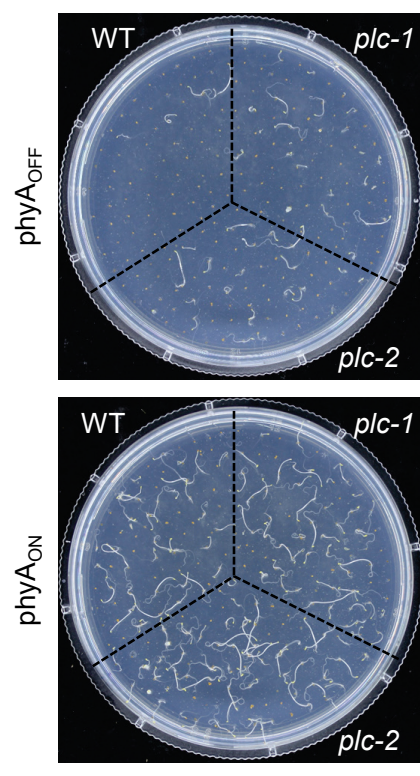

B
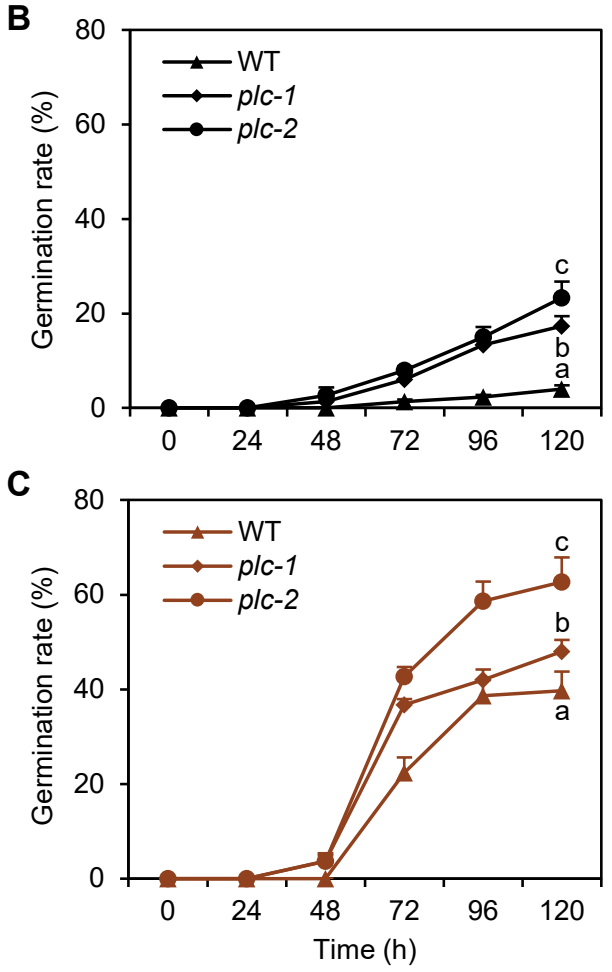

E
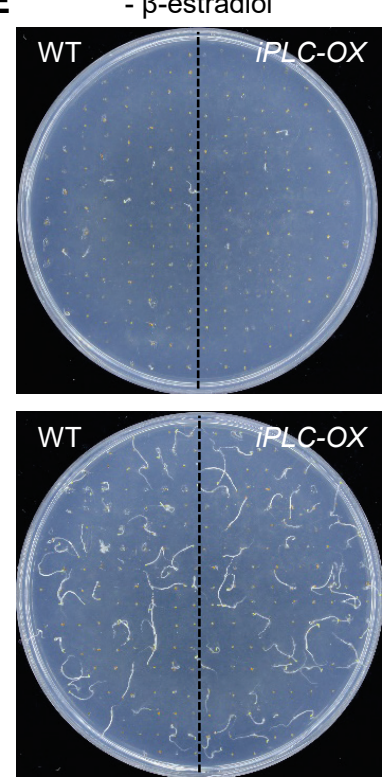

$+\beta$-estradiol
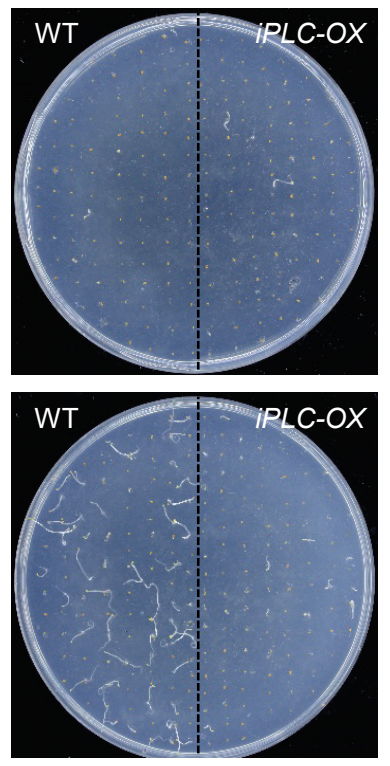

D

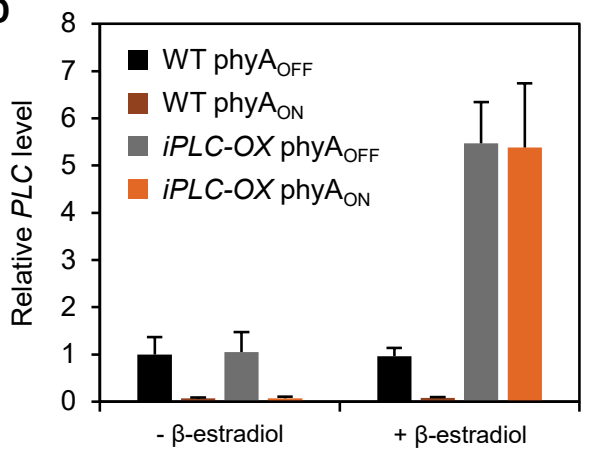

$\mathbf{F}$

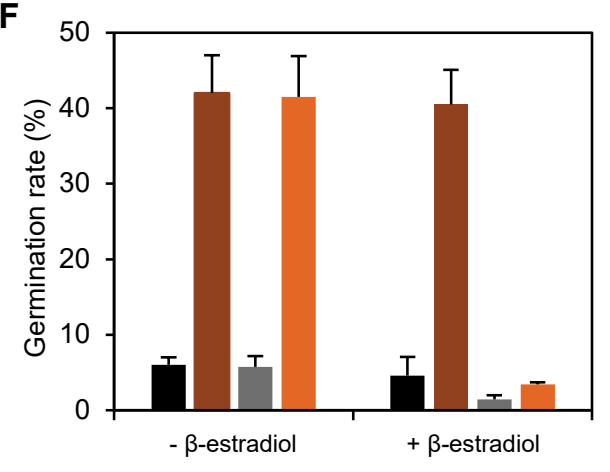

Figure 2. PLC Negatively Regulates Germination.

(A) Representative plates showing germination state of the wild type and p/c seeds in phy $A_{O F F}$ (top) and phyA $\mathrm{ON}_{\mathrm{N}}$ (bottom). (B-C) Quantification of the germination rate over the time course of phy $\mathrm{A}_{\mathrm{OFF}}$ $(B)$ and phyA $A_{O N}(C)$. Data are mean $\pm S D(n=3)$. Different letters represent genotypes with significant differences at $120 \mathrm{~h}$ (ANOVA, $p<0.05$ ). (D) RT-qPCR analysis of relative PLC transcript level in the indicated genotypes without and with the application of $\beta$-estradiol. Data are mean \pm SD $(n=3)$. (E) Representative plates showing germination state of the wild type and iPLC-OX seeds in phy $A_{O F F}$ and phyA $A_{O N}$ under the indicated treatments. $(F)$ Quantification of germination rates of the wild type and $P L C-O X$ seeds. Data are mean $\pm S D(n=3)$. 


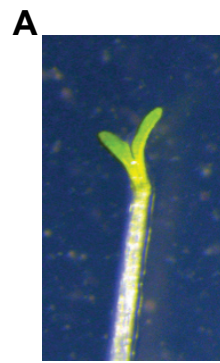

WT
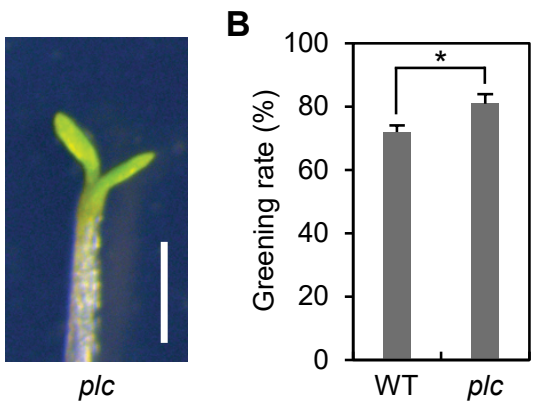

C

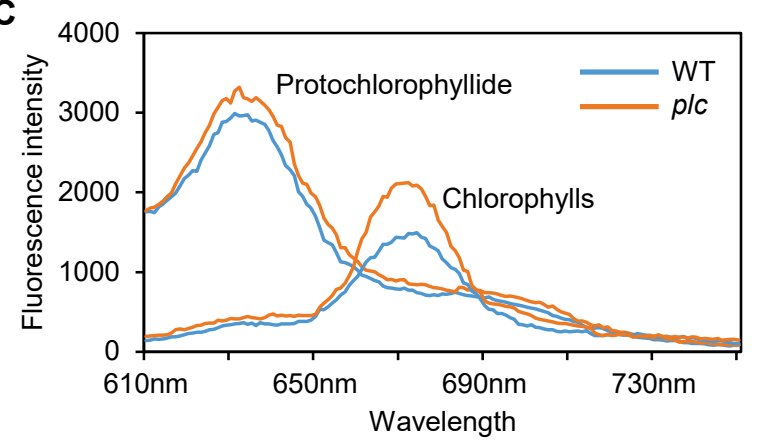

Figure 3. PLC Negatively Regulates Seedling Greening.

(A) Representative wild type and p/c seedlings that were grown in the dark for $96 \mathrm{~h}$ and then exposed to continuous white light for $24 \mathrm{~h}$. Bar, $1 \mathrm{~mm}$. (B) Quantified greening rate. Data are mean $\pm \mathrm{SD}(\mathrm{n}=50) .{ }^{*}, p<0.05$ by Student's $t$ test. (C) Comparison of pigment profile in the p/c and wild type seedlings. Etiolated seedlings grown in the dark for $96 \mathrm{~h}$ were assayed for protochlorophyllide by spectral analysis. Chlorophylls were assayed in etiolated seedlings exposed to white light for $24 \mathrm{~h}$. 
A

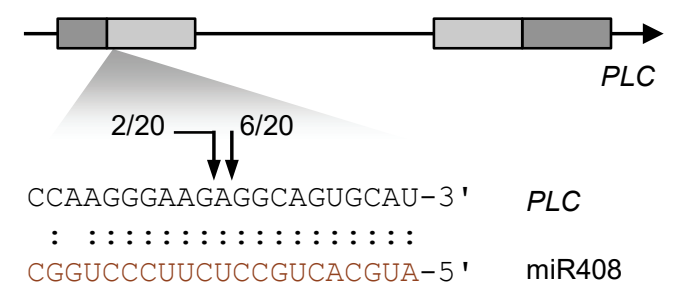

B

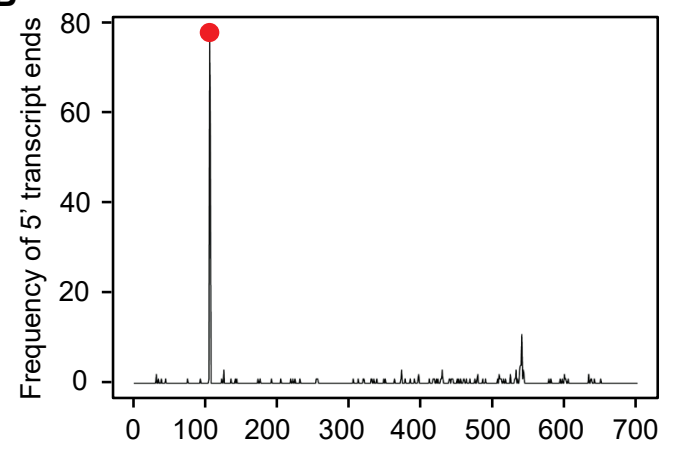

C

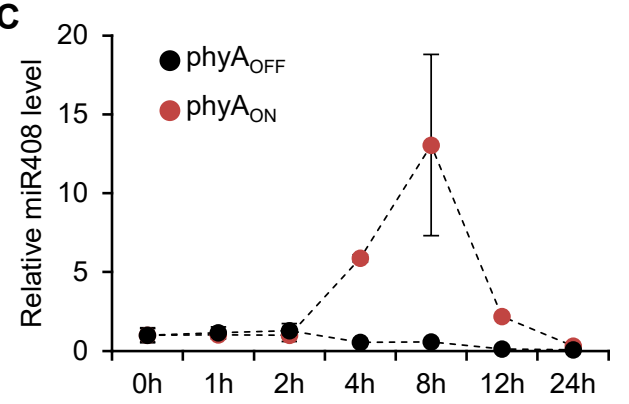

D

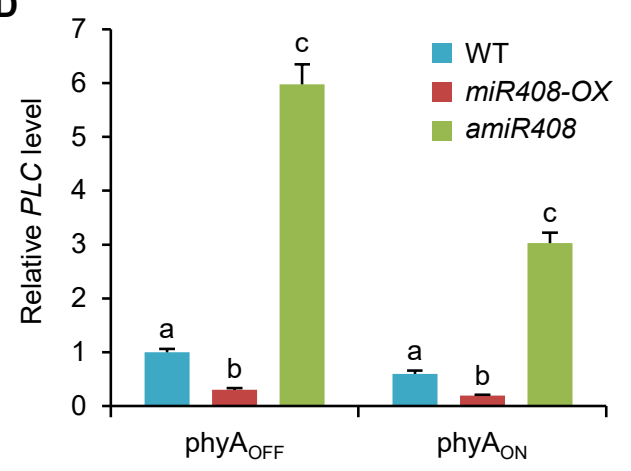

Figure 4. miR408 Represses PLC Expression during Germination.

(A) Confirmation of miR408 targeting on PLC in the seed by RNA-ligation based amplification of cDNA ends. Gene structure of $P L C$ is shown on top. Base pairing between miR408 and $P L C$ is shown on bottom. Arrows mark the detected transcript ends along with frequency of the corresponding clones. (B) Degradome sequencing data supporting miR408-guided cleavage of PLC. Frequency of the sequenced 5' ends is plotted against nucleotide position in the PLC transcript. Red dot indicates position of the miR408 recognition site. (C) RT-qPCR analysis of relative miR408 levels over the time course of phyA $A_{O F F}$ and phyA $A_{O N}$. Data are mean $\pm S D(n=3)$. (D) Relative PLC transcript levels in seeds of the indicated genotypes under phyA $\mathrm{OFF}_{\text {and }}$ phyA $\mathrm{ON}_{\mathrm{ON}}$. Data are means $\pm S D(n=3)$. Different letters denote genotypes with significant difference (ANOVA, $p<0.05$ ). 
A

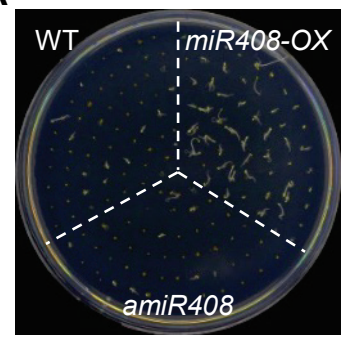

phyA $\mathrm{AFF}_{\mathrm{O}}$

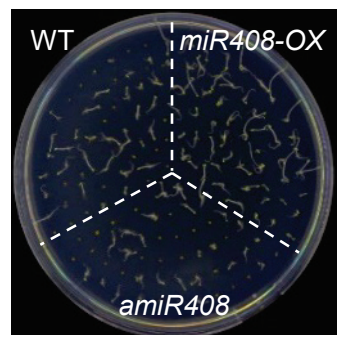

phyA $_{O N}$

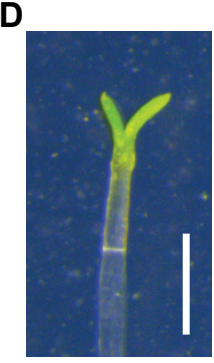

WT

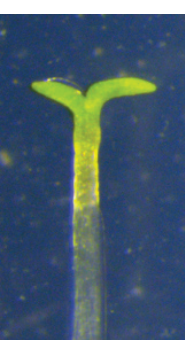

$\operatorname{miR} 408-0 X$

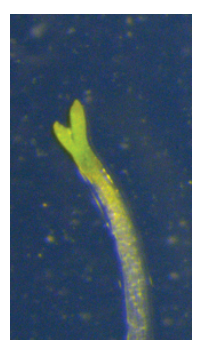

amiR408
B

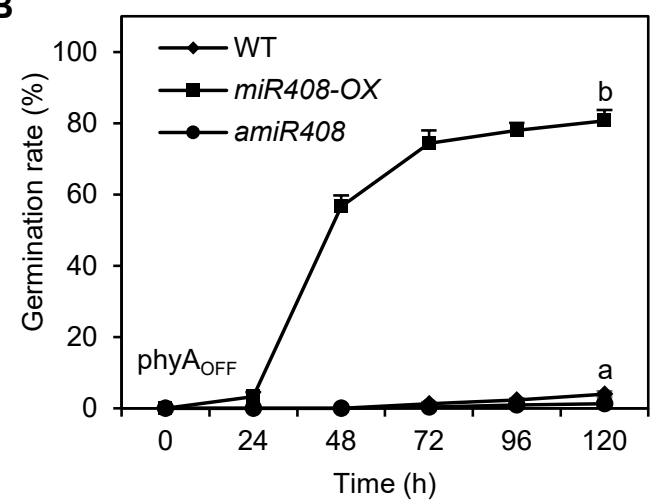

C

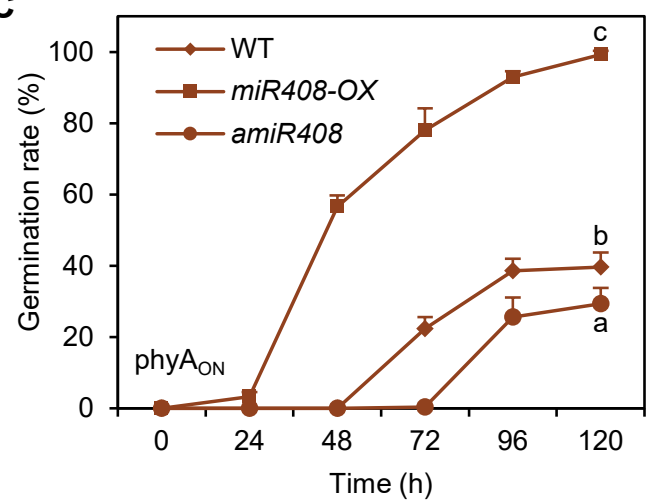

E
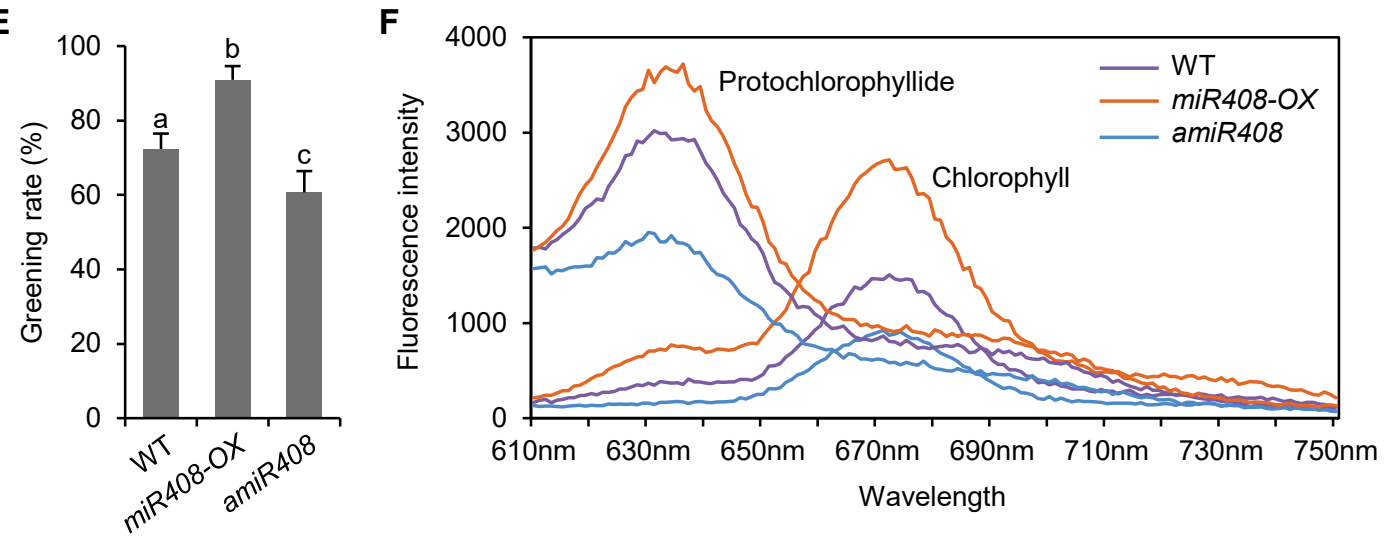

Figure 5. miR408 Promotes Seed Germination and Seedling Greening.

(A) Representative plates showing germination state of the indicated genotypes under phy $\mathrm{A}_{\mathrm{OFF}}$ (left) and phyA $\mathrm{ON}_{\mathrm{N}}$ (right). (B-C) Quantification of germination rate of the indicated genotypes over the time course of phy $A_{O F F}(B)$ and phyA $A_{O N}(C)$. Data are mean $\pm S D(n=3)$. Different letters denote genotypes with significant differences at $120 \mathrm{~h}$ (ANOVA, $p<0.05$ ). (D) Representative etiolated seedlings of the indicated genotypes exposed to white light for $24 \mathrm{~h}$. Bar, $1 \mathrm{~mm}$. (E) Quantified greening rate. Data are mean $\pm S D(n=50)$. Different letters represent significant differences (ANOVA, $p<0.05$ ). (F) Comparison of pigment profile in the indicated genotypes. Protochlorophyllide and chlorophylls were analyzed in etiolated seedlings and etiolated seedlings exposed to white light for $24 \mathrm{~h}$, respectively. 
A
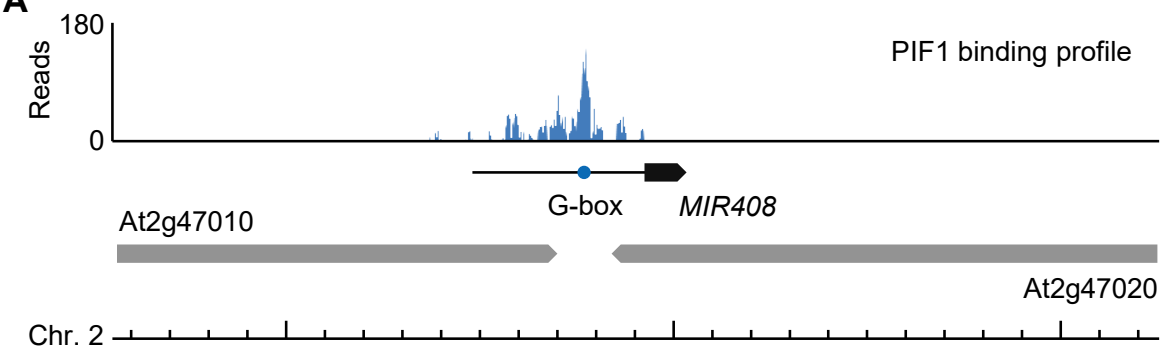

C

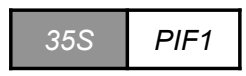

Effecter

\begin{tabular}{|l|l|l|l|}
\hline $35 S$ & REN & pMIR408 & LUC \\
\hline
\end{tabular}

Dual luciferase reporter

E

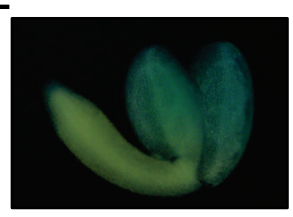

pMIR408:GUSNT

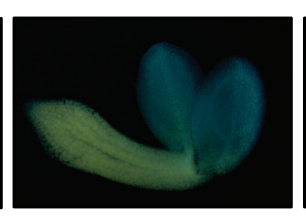

pMIR408:GUS/pif1
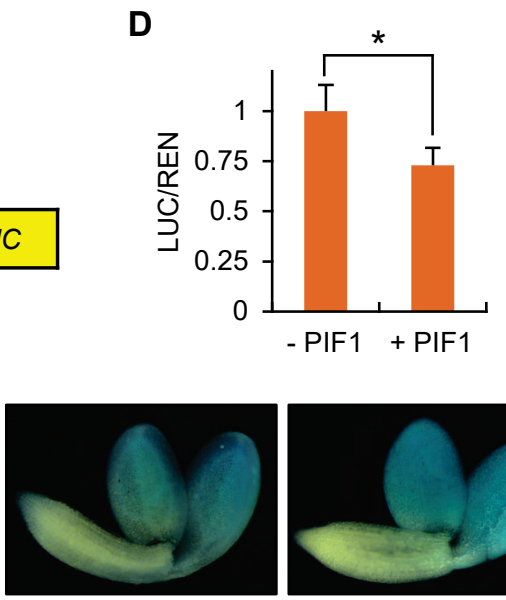

PMIR408:GUSNT $\quad$ PMIR408:GUS/pif1

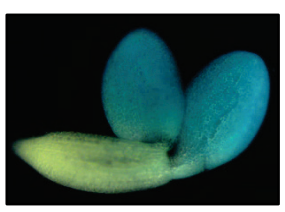

phyA $_{\mathrm{ON}}$

PhyA ${ }_{\text {OFF }}$

B
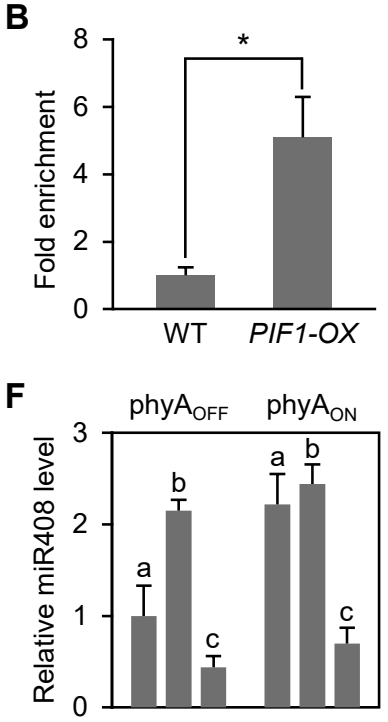

G

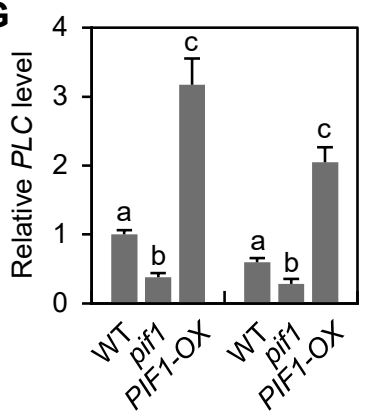

Figure 6. PIF1 Suppresses miR408 Expression by Binding to the MIR408 Promoter.

(A) PIF1 occupancy profile at the MIR408 locus. Significantly enriched PIF1 ChIP-sequencing reads were obtained from Pfeiffer et al. (2014) and mapped onto the Arabidopsis genome coordinates. Loci are represented by block arrows. The blue circle marks the G-box (CACGTG) in the MIR408 promoter (horizontal line). (B) ChIP-qPCR confirming PIF1 binding to the MIR408 promoter. An anti-MYC antibody was used to precipitate chromatin from PIF1-OX and wild type seeds. Enrichment of PIF1 binding was determined by qPCR analysis. Data are mean $\pm \mathrm{SD}(\mathrm{n}=3) .{ }^{*}, p<0.05$ by Student's $t$ test. (C) Transient dual luciferase assay showing PIF1 repression of MIR408. The pMIR408:LUC reporter concatenated to 35S:REN was used to transform tobacco protoplasts with either the empty vector (PIF1) or a PIF1-expressing construct (+ PIF1). (D) Quantification of the LUC/REN luminescence ratio. Data are mean $\pm \mathrm{SD}(\mathrm{n}=3) .{ }^{*}, p<0.05$ by Student's $t$ test. (E) Comparison of GUS activity in transgenic seed expressing pMIR408:GUS in the wild type or pif1 background in phyA $\mathrm{OFF}_{\text {af }}$ and phyA $\mathrm{ON}_{\mathrm{ON}}$. Bar, $500 \mu \mathrm{m}$. (F-G) RT-qPCR analysis of relative miR408 (F) and PLC (G) transcript levels. Data are mean $\pm S D(n=3)$. Different letters denote genotypes with significant differences (ANOVA, $p<0.05$ ). 
A

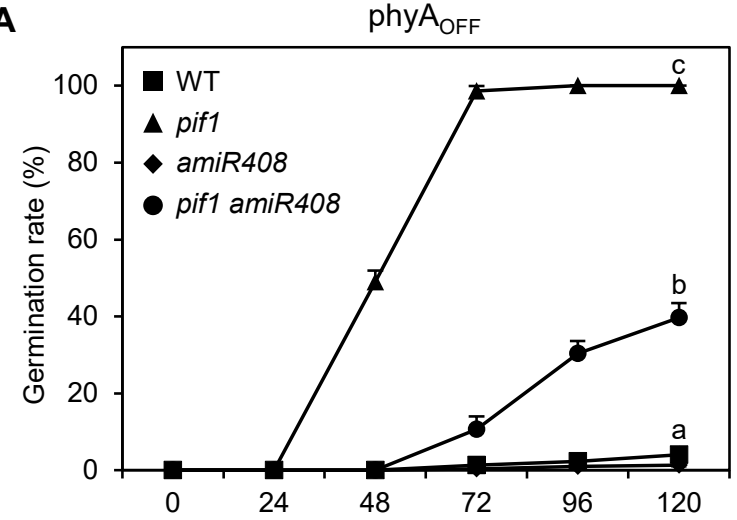

B

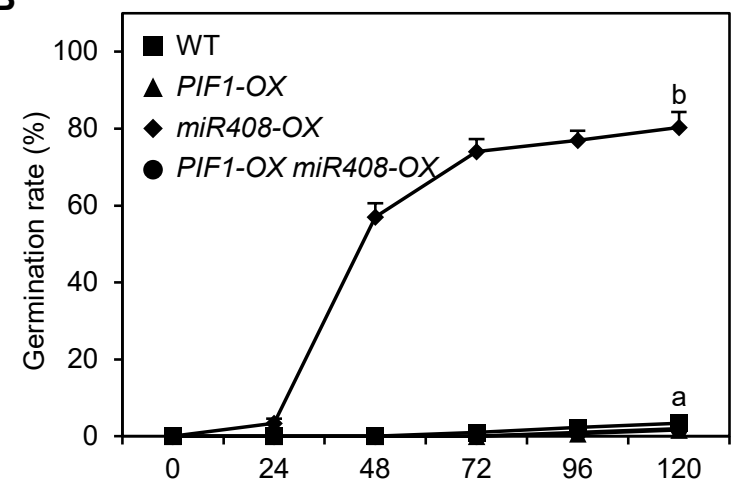

C

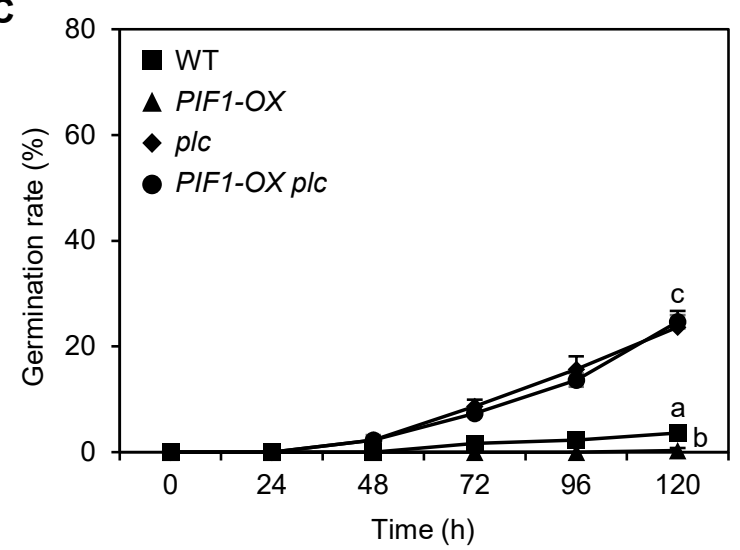

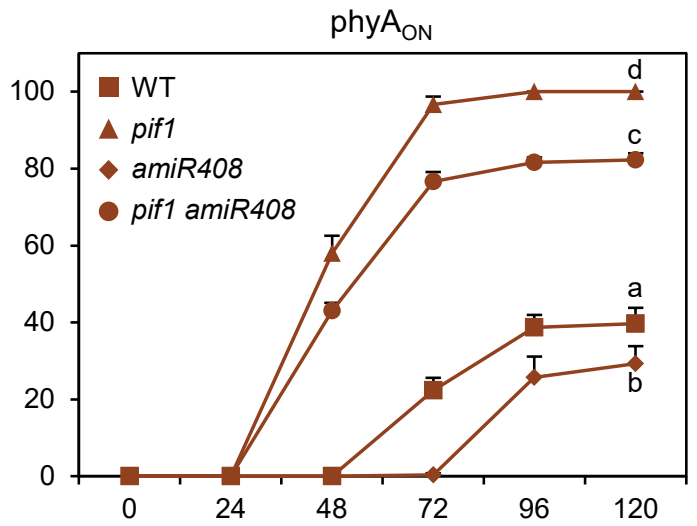
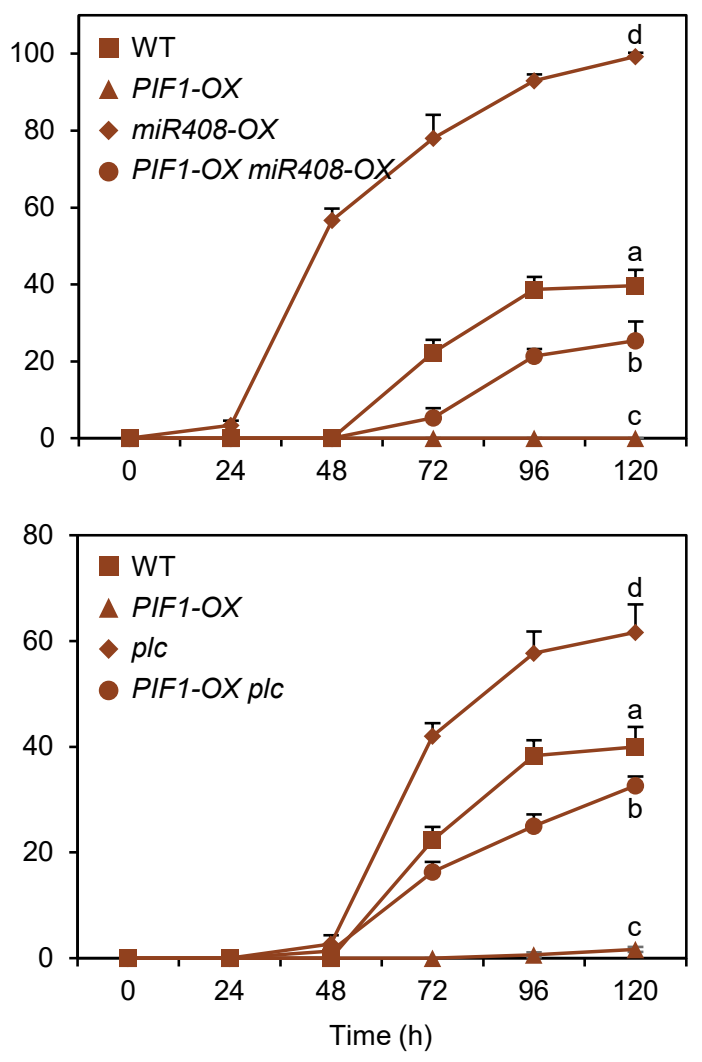

Figure 7. Genetic Analysis of the PIF1-MIR408-PLC Pathway.

(A) The amiR408 line was crossed with pif1 to generate the pif1 amiR408 double mutant. Seeds from these lines and the wild type were assayed for germination rates over the time course of

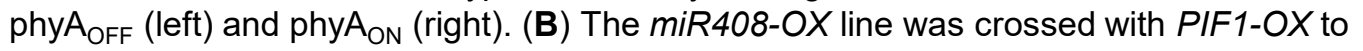
generate the PIF1-OX miR408-OX double over-expression line. Seeds from these lines and the wild type were assayed for germination rates in phyA $\mathrm{OFF}_{\text {and }}$ any $\mathrm{A}_{\mathrm{ON}}$. (C) Comparison of germination rates of the PIF1-OX, plc, and PIF1-OX plc seeds. Data are all means \pm SD $(n=3)$. Different letters denote genotypes with significant differences at $120 \mathrm{~h}$ (ANOVA, $p<0.05$ ). 
A

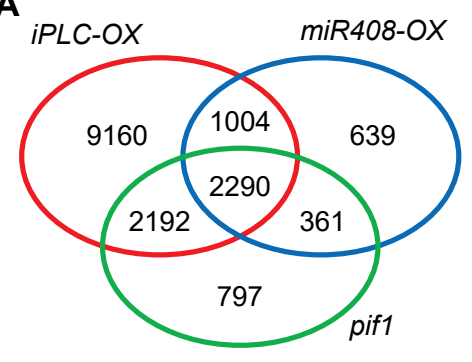

B
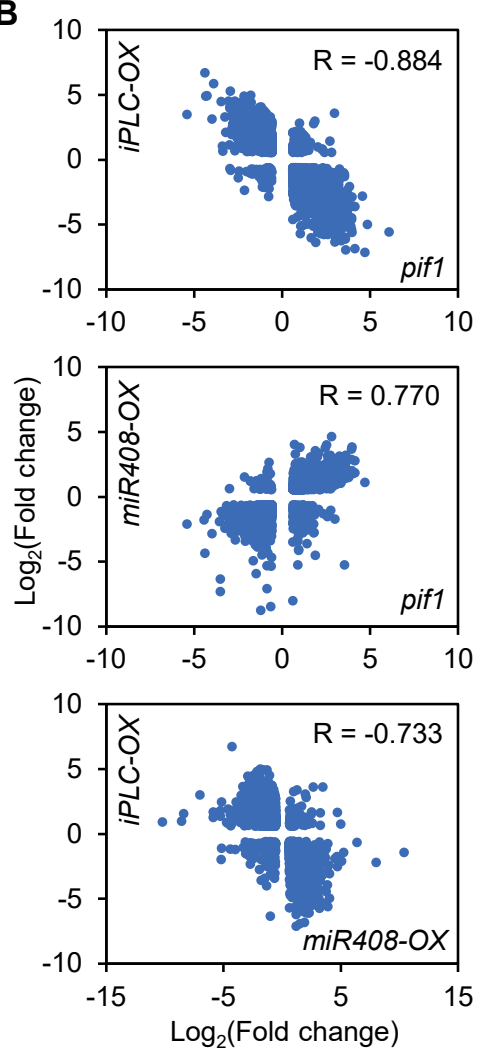

C

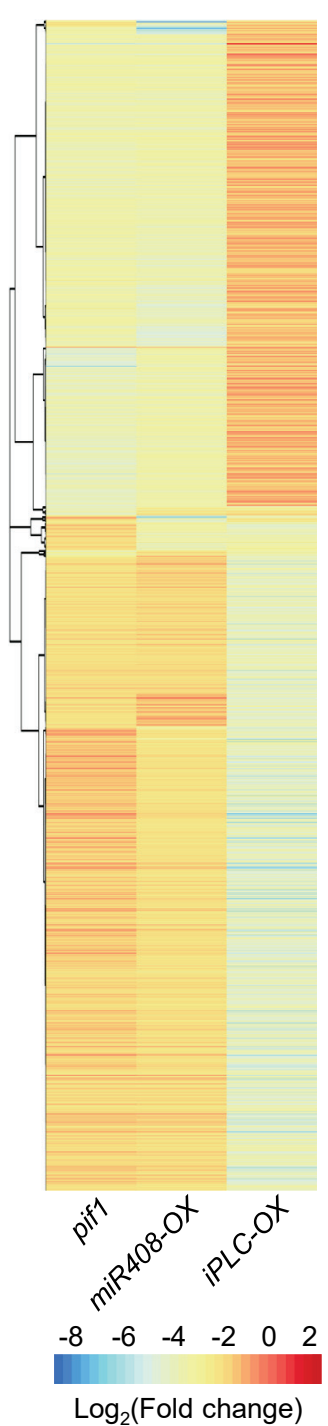

D

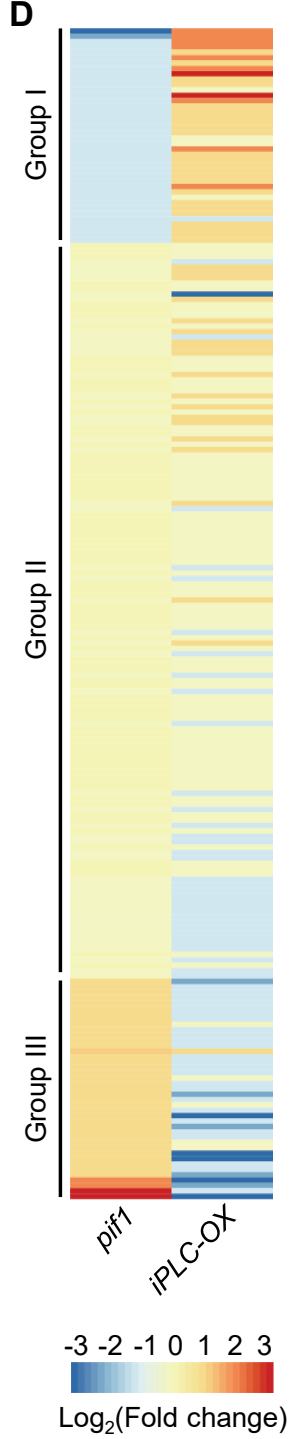

E
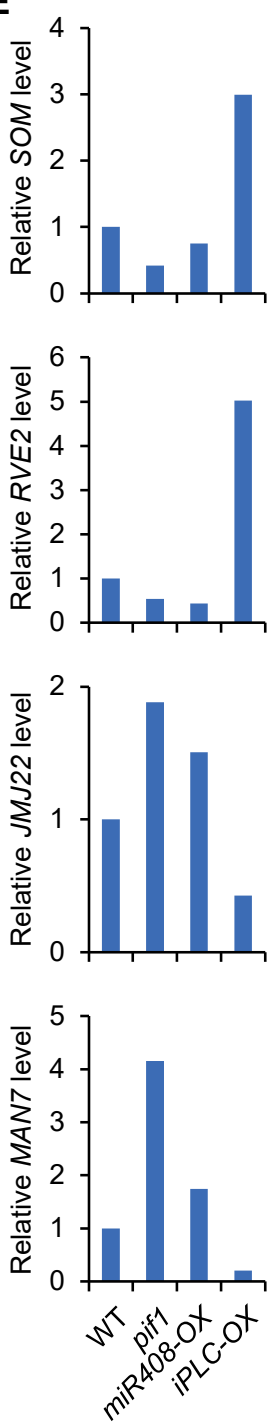

\section{Figure 8. Transcriptomic Analysis of the PIF1-MIR408-PLC Pathway.}

(A) Venn diagram showing the relationships of PIF1, MIR408, and PLC regulated genes. Differentially expressed genes were identified from RNA-sequencing analysis of pif1, miR408-OX, and iPLC-OX seeds against the respective controls. (B) Scatterplots showing pairwise correlation of the relative expression levels of the three sets of coregulated genes in pif1, miR408-OX, and iPLC-OX against the respective controls. R, Pearson correlation coefficient. (C) Hierarchical clustering of the 2,290 genes differentially expressed in pif1, miR408-OX, and iPLC-OX against the respective controls. Colors represent the $\mathrm{Log}_{2}$ transformed fold change. (D) Clustering analysis of the 218 genes associated with the GO term "seed germination" (GO:0009845). The genes were divided in three grouped based relative expression level in pif1 against the wild type. Group I, repressed in pif1; Group II, not differentially expressed; Group III, induced in pif1. (E) Expression pattern of representative Group I (SOM and RVE2) and Group III (JMJ22 and MAN7) genes in the indicted RNA-sequencing datasets. 
A

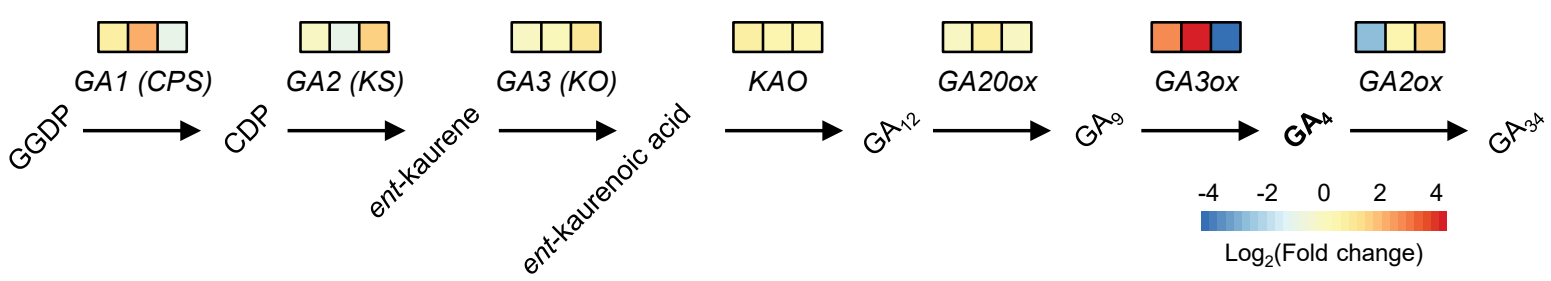

B

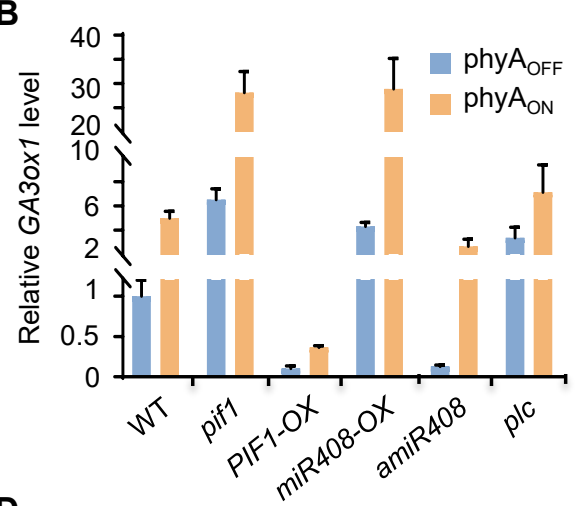

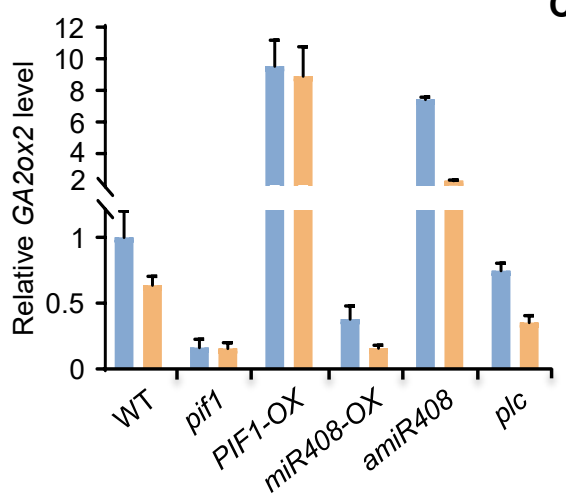

C

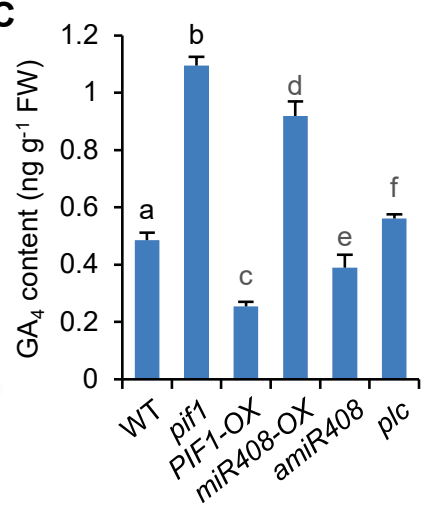

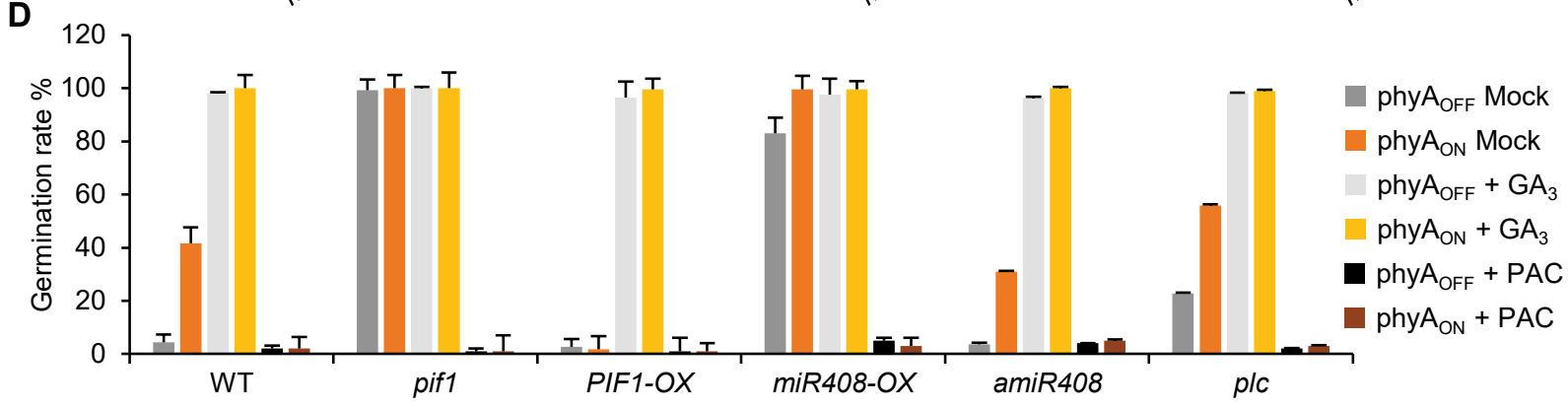

Figure 9. The PIF1-MIR408-PLC Pathway Modulates GA Biosynthesis.

(A) Diagram of a simplified GA biosynthesis pathway illustrating genes influenced by the PIF1-MIR408PLC pathway. Genes associated with the individual biosynthesis steps are shown on top of the arrows. Colored boxes indicate relative expression levels of the corresponding gene in pif1, miR408-OX, and iPLC-OX against the respective controls. (B) RT-qPCR analysis of relative transcript level of the GA3ox1 and GA2ox2 genes in the indicated seeds under phyA $\mathrm{OFF}_{\text {and phyA }}$ aN. Data are mean $\pm S D(n=3)$. (C) Quantification of endogenous $\mathrm{GA}_{4}$ levels in imbibed seed of the indicated genotypes. Data are mean \pm $\mathrm{SD}(\mathrm{n}=3)$. Different letters denote genotypes with significant differences (ANOVA, $p<0.05)$. (D) Germination rates of the indicated seeds in phy $A_{O F F}$ and phyA $A_{O N}$ with different treatments. Mock, no chemical treatment; $\mathrm{GA}_{3}, 10 \mu \mathrm{M} \mathrm{GA}$; PAC, $100 \mu \mathrm{M}$ paclobutrazol. Data are mean $\pm \mathrm{SD}(\mathrm{n}=3)$. 
A

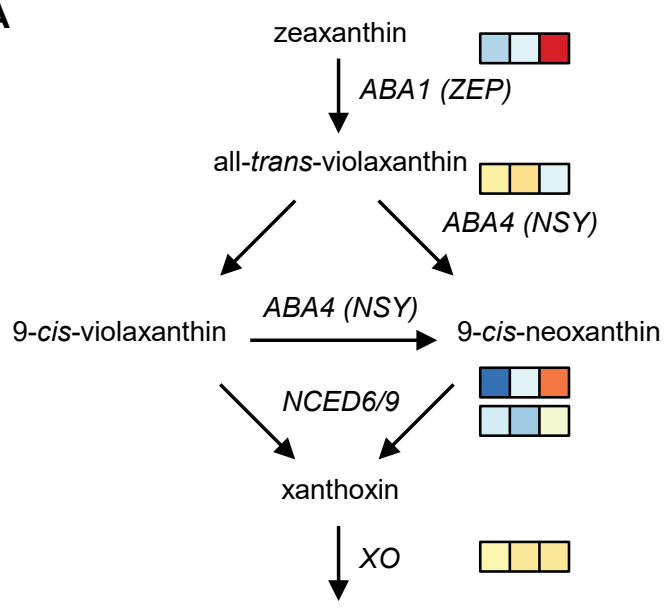

abscisic aldehyde

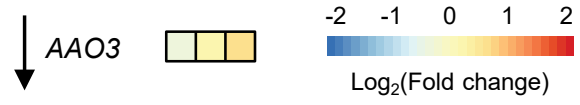

B

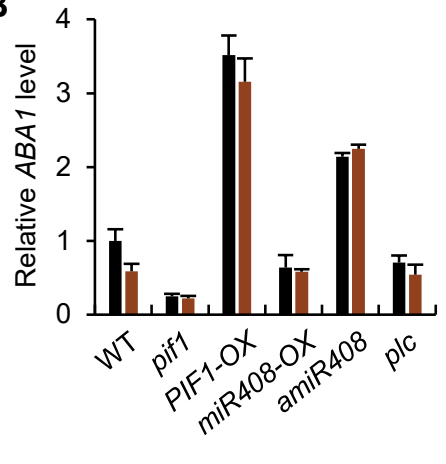

Log $_{2}($ Fold change)

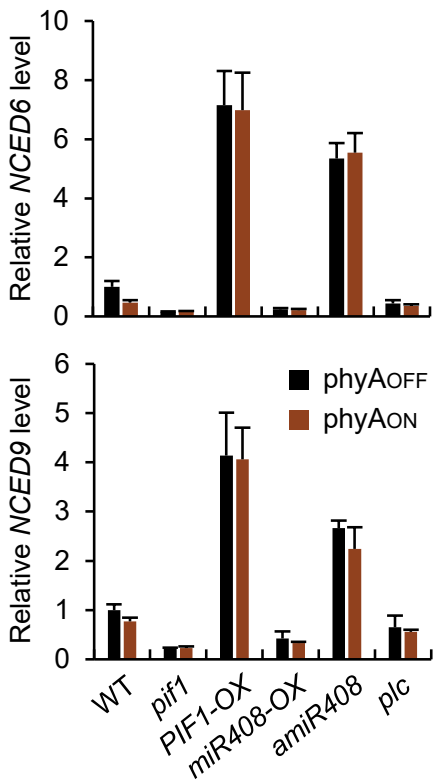

C

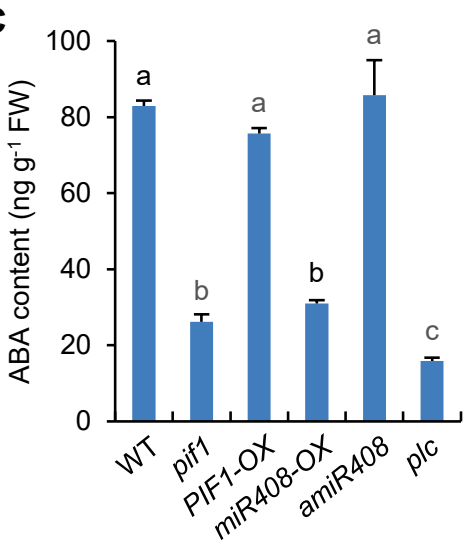

D

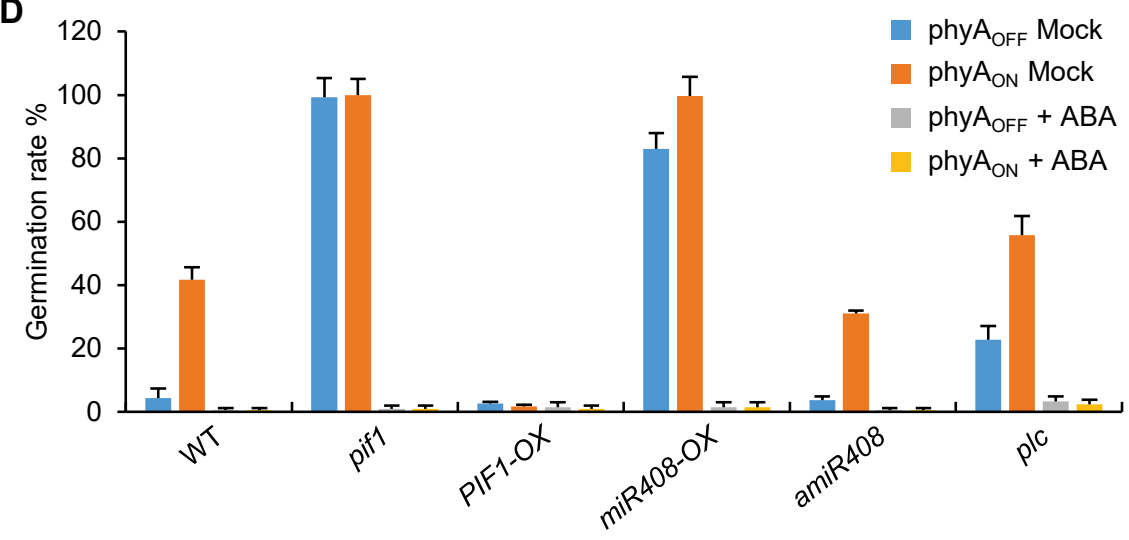

Figure 10. The PIF1-MIR408-PLC Pathway Regulates ABA Biosynthesis.

(A) Diagram of a simplified ABA biosynthesis pathway illustrating genes influenced by the PIF1-MIR408$P L C$ pathway. Colored boxes indicate relative expression levels of the corresponding gene in pif1, miR408-OX, and iPLC-OX against the respective controls. (B) RT-qPCR analysis of the relative transcript levels of ABA metabolic genes ABA1, NCED6, and NCED9 in the indicated seeds. Data are mean \pm SD $(n=3)$. (C) Quantification of endogenous ABA level in the indicated seeds. Data are mean $\pm S D(n=3)$. Different letters denote groups with significant differences (ANOVA, $p<0.05$ ). (D) Germination rate of the indicated seeds in phyA $A_{O F F}$ and phyA $A_{O N}$ with or without the application of $5 \mu \mathrm{M} A B A$. 


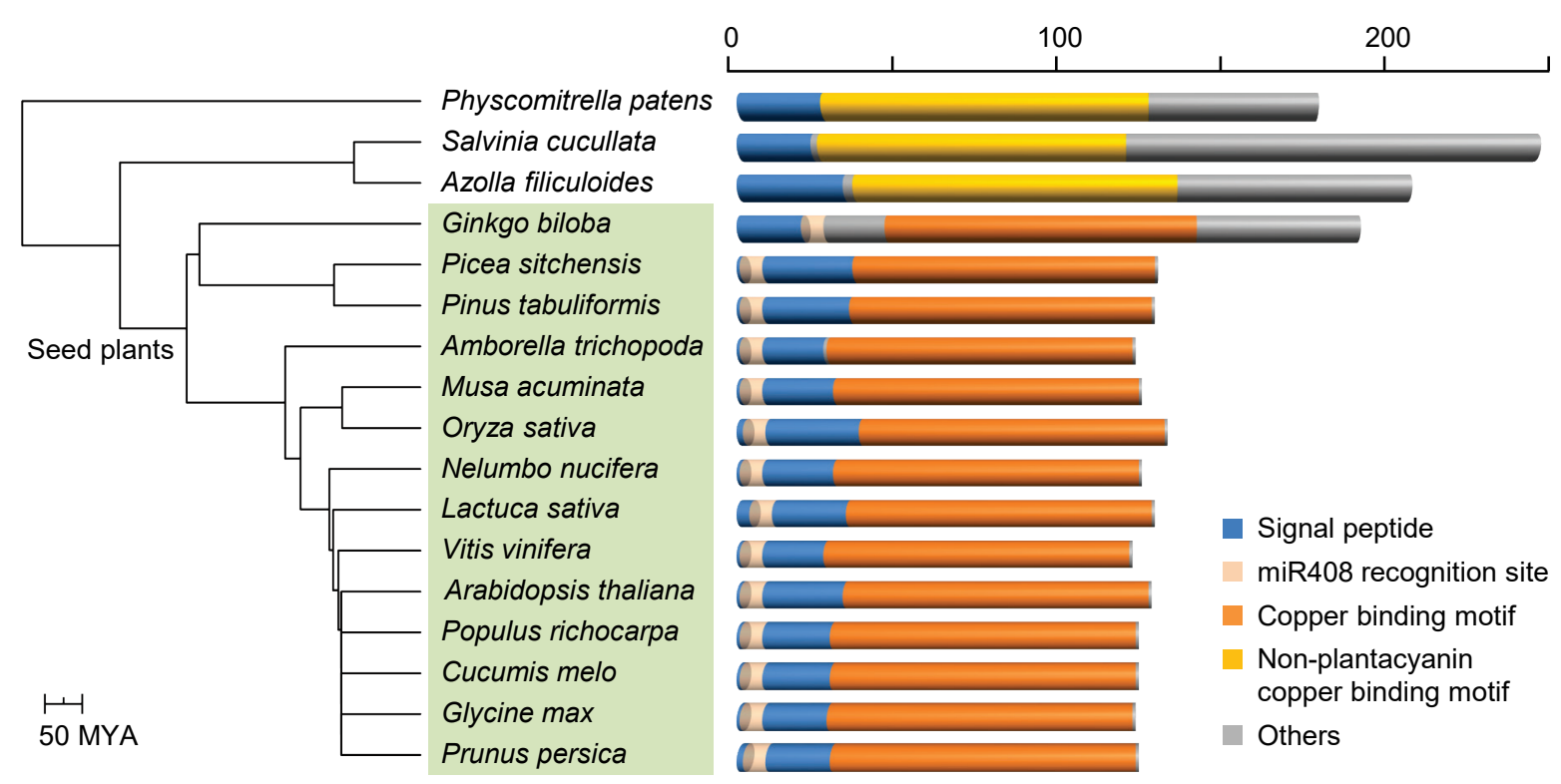

Figure 11. PLC Exhibits Features Conserved in Seed Plants.

Comparison of PLC and related blue copper proteins in representative land plants. Shown on the left is a species tree. Branch length reflects evolutionary divergence time in millions of years inferred from TimeTree. Species with identified PLCs are shaded in green. Domains are shown with different colors on the right. Scale represents accumulative number of amino acids. 


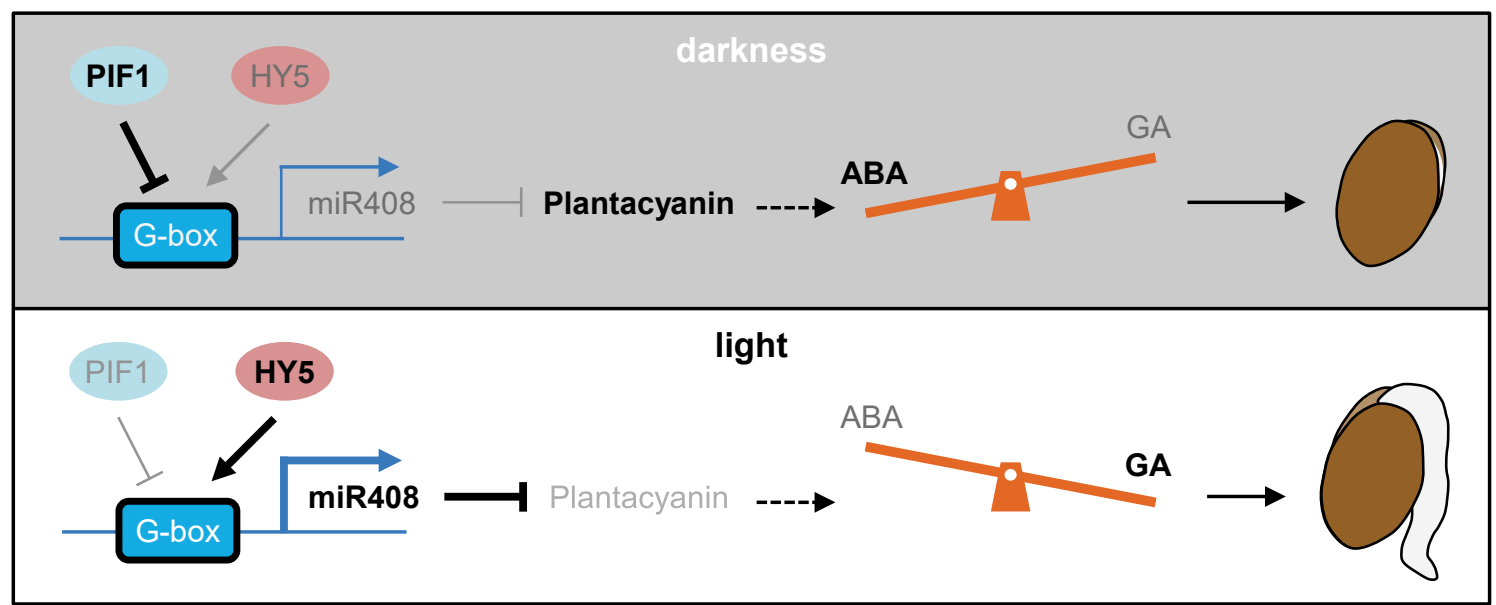

Figure 12. Working Model of Light-dependent Seed Germination Mediated by PLC.

The PIF1/HY5-miR408 module is critical for regulating PLC abundance in light-induced seed germination. Both PIF1 and HY5 can bind to the G-box cis-element in the MIR408 promoter and thereby modulate cellular miR408 level. In darkness, stabilized PIF1 is the predominant regulator leading to transcriptional repression of miR408, which allows PLC to accumulate. Upon light irradiation, HY5 activation and PIF degradation leads to transcriptional de-repression of miR408, which in turn silences PLC. Removal of storage vacuole located PLC facilitates establishing the high-GA-low-ABA hormonal profile that eventually sets germination in motion and promotes postgerminative growth in light. 


\section{Parsed Citations}

Abdel-Ghany, S.E., and Pilon, M. (2008). MicroRNA-mediated systemic down-regulation of copper protein expression in response to low copper availability in Arabidopsis. J Biol Chem. 283: 15932-15945.

Pubmed: Author and Title

Google Scholar: Author Only Title Only Author and Title

Almagro Armenteros, J.J., Tsirigos, K.D., Sønderby, C.K., Petersen, T.N., Winther, O., Brunak, S., von Heijne, G., and Nielsen, H. (2019). SignalP 5.0 improves signal peptide predictions using deep neural networks. Nat Biotechnol. 37: 420-423.

Pubmed: Author and Title

Google Scholar: Author Only Title Only Author and Title

Angelovici, R., Galili, G., Fernie, AR., and Fait, A (2010). Seed desiccation: a bridge between maturation and germination. Trends Plant Sci. 15: 211-218.

Pubmed: Author and Title

Google Scholar: Author Only Title Only Author and Title

Bernala, M., Ramiroa, M.V., Casesb, R., Picorela, R., and Yruela, I. (2006). Excess copper effect on growth, chloroplast ultrastructure, oxygen-evolution activity and chlorophyll fluorescence in Glycine max cell suspensions. Physiologia Plantarum 127: 312-325.

Pubmed: Author and Title

Google Scholar: Author Only Title Only Author and Title

Bewley, J.D. (1997). Seed germination and dormancy. Plant Cell 9: 1055-1066.

Pubmed: Author and Title

Google Scholar: Author Only Title Only Author and Title

Burkhead, J.L., Reynolds, K.A, Abdel-Ghany, S.E., Cohu, C.M., and Pilon, M. (2009). Copper homeostasis. New Phytol. 182: 799-816.

Pubmed: Author and Title

Google Scholar: Author Only Title Only Author and Title

Castillon, A, Shen, H., and Huq, E. (2007). Phytochrome Interacting Factors: Central players in phytochrome-mediated light signaling networks. Trends Plant Sci. 12: 514-521.

Pubmed: Author and Title

Google Scholar: Author Only Title Only Author and Title

Chen, D., Xu, G., Tang, W., Jing, Y., Ji, Q., Fei, Z, and Lin, R. (2013). Antagonistic basic helix-loop-helix/bZP transcription factors form transcriptional modules that integrate light and reactive oxygen species signaling in Arabidopsis. Plant Cell 25: 1657-1673.

Pubmed: Author and Title

Google Scholar: Author Only Title Only Author and Title

Cho, J., Ryu, J., Jeong, Y., Park, J., Song, J., Amasino, R.M., Noh, B., and Noh, Y. (2012). Control of seed germination by light-induced histone arginine demethylation activity. Dev Cell 22: 736-748.

Pubmed: Author and Title

Google Scholar: Author Only Title Only Author and Title

Dai, X., Zhuang, Z, and Zhao, P.X. (2018). psRNATarget: a plant small RNA target analysis server. Nucleic Acids Res. 46: W49-W54.

Pubmed: Author and Title

Google Scholar: Author Only Title Only Author and Title

De Rienzo, F., Gabdoulline, R.R., Menziani, M.C., and Wade, R.C. (2000). Blue copper proteins: a comparative analysis of their molecular interaction properties. Protein Sci. 9: 1439-1454.

Pubmed: Author and Title

Google Scholar: Author Only Title Only Author and Title

Dong, J., Kim S.T., and Lord, E.M. (2005) Plantacyanin plays a role in reproduction in Arabidopsis. Plant Physiol. 138: 778-789.

Pubmed: Author and Title

Google Scholar: Author Only Title Only Author and Title

Eroglu, S., Giehl, R.F.H., Meier, B., Takahashi, M., Terada, Y., Ignatyev, K., Andresen, E., Küpper, H., Peiter, E., and von Wirén, N. (2017). Metal tolerance protein 8 mediates manganese homeostasis and iron reallocation during seed development and germination. Plant Physiol. 174: 1633-1647.

Pubmed: Author and Title

Google Scholar: Author Only Title Only Author and Title

Feng, H., Zhang, Q., Wang, Q., Wang, X., Liu, J., Li, M., Huang, L., and Kang Z (2013). Target of tae-miR408, a chemocyanin-like protein gene (TaCLP1), plays positive roles in wheat response to high-salinity, heavy cupric stress and stripe rust. Plant Mol Biol. 83: 433-443. Pubmed: Author and Title

Google Scholar: Author Only Title Only Author and Title

Finch-Savage, W.E., and Leubner-Metzger, G. (2006). Seed dormancy and the control of germination. New Phytol. 171: 501-523.

Pubmed: Author and Title

Google Scholar: Author Only Title Only Author and Title

Finkelstein, R., Reeves, W., Ariizumi, T., and Steber, C. (2008). Molecular aspects of seed dormancy. Annu Rev Plant Biol. 59: 387-415. 
Pubmed: Author and Title

Google Scholar: Author Only Title Only Author and Title

Fu, J., Chu, J., Sun, X., Wang, J., and Yan, C. (2012). Simple, rapid, and simultaneous assay of multiple carboxyl containing phytohormones in wounded tomatoes by UPLC-MS/MS using single SPE purification and isotope dilution. Anal Sci. 28: $1081-1087$.

Pubmed: Author and Title

Google Scholar: Author Only Title Only Author and Title

Giri, AV., Anishetty, S., and Gautam, P. (2004). Functionally specified protein signatures distinctive for each of the different blue copper proteins. BMC Bioinformatics 9: 127.

Pubmed: Author and Title

Google Scholar: Author Only Title Only Author and Title

Guo, Z, Kuang, Z, Wang, Y., Zhao, Y., Tao, Y., Cheng, C., Yang, J., Lu, X., Hao, C., Wang, T., Cao, X., Wei, J., Li, L., and Yang, X. (2020). PmiREN: Acomprehensive encyclopedia of plant miRNAs. Nucleic Acids Res. 48: D1114-D1121.

Pubmed: Author and Title

Google Scholar: Author Only Title Only Author and Title

Gupta, AS., Heinen, J.L., Holaday, AS., Burke, J.J., and Alen, R.D. (1993). Increased resistance to oxidative stress in transgenic plants that overexpress chloroplastic Cu/Zn superoxide dismutase. Proc Natl Acad Sci U S A 90: 1629-1633.

Pubmed: Author and Title

Google Scholar: Author Only Title Only Author and Title

Guss, J.M., Merritt, E.A, Phizackerley, R.P., Hedman, B., Murata, M., Hodgson, K.O., and Freeman, H.C. (1998). Phase determination by multiple-wavelength x-ray diffraction: crystal structure of a basic blue copper protein from cucumbers. Science 241: 806-811.

Pubmed: Author and Title

Google Scholar: Author Only Title Only Author and Title

Han, X., Chang, X., Zhang, Z, Chen, H., He, H., Zhong, B., and Deng, X.W. (2019). Origin and evolution of core components responsible for monitoring light environment changes during plant terrestrialization. Mol Plant 12: 847-862.

Pubmed: Author and Title

Google Scholar: Author Only Title Only Author and Title

Hellens, R.P., Alan, AC., Friel, E.N., Bolitho, K., Grafton, K., Templeton, M.D., Karunairetnam, S., Gleave, AP., and Laing, W.A (2005). Transient expression vectors for functional genomics, quantification of promoter activity and RNA silencing in plants. Plant Methods 1 : 13.

Pubmed: Author and Title

Google Scholar: Author Only Title Only Author and Title

Iglesias-Fernández, R., Rodríguez-Gacio, M.C., Barrero-Sicilia, C., Carbonero, P., and Matilla, AJ. (2011) Three endo- $\beta$-mannanase genes expressed in the micropilar endosperm and in the radicle influence germination of Arabidopsis thaliana seeds. Planta 233: 2536.

Pubmed: Author and Title

Google Scholar: Author Only Title Only Author and Title

Jiang, Z, Xu, G., Jing, Y., Tang, W., and Lin, R. (2016). Phytochrome B and REVEILLE1/2-mediated signaling controls seed dormancy and germination in Arabidopsis. Nat Commun. 7: 12377.

Pubmed: Author and Title

Google Scholar: Author Only Title Only Author and Title

Jung, H.W., and Hwang, B.K. (2000). Isolation, partial sequencing, and expression of pathogenesis-related cDNAgenes from pepper leaves infected by Xanthomonas Campestris Pv. Vesicatoria. Mol Plant Microbe Interact. 13: 136-142.

Pubmed: Author and Title

Google Scholar: Author Only Title Only Author and Title

Kallioo, P., and Piiroinen, P. (1959). Effect of gibberellin on the termination of dormancy in some seeds. Nature 183: $1830-1831$.

Pubmed: Author and Title

Google Scholar: Author Only Title Only Author and Title

Kim, D.H., Yamaguchi, S., Lim, S., Oh, E., Park, J., Hanada, A, Kamiya, Y., and Choi, G. (2008). SOMNUS, a CCCH-type zinc finger protein in Arabidopsis, negatively regulates light-dependent seed germination downstream of PIL5. Plant Cell 20: $1260-1277$.

Pubmed: Author and Title

Google Scholar: Author Only Title Only Author and Title

Kim, S., Mollet, J.C., Dong, J., Zhang, K., Park, S.Y., and Lord, E.M. (2003) Chemocyanin, a small basic protein from the lily stigma, induces pollen tube chemotropism. Proc Natl Acad Sci U S A 100: 16125-16130

Pubmed: Author and Title

Google Scholar: Author Only Title Only Author and Title

Kim, S.A, Punshon, T., Lanzirotti, A, Li, L., Alonso, J.M., Ecker, J.R., Kaplan, J., and Guerinot, M.L. (2006). Localization of iron in Arabidopsis seed requires the vacuolar membrane transporter VT1. Science 314: 1295-1298.

Pubmed: Author and Title

Google Scholar: Author Only Title Only Author and Title

Klaumann, S., Nickolaus, S.D., Fürst, S.H., Starck, S., Schneider, S., Ekkehard, Neuhaus, H., and Trentmann, O. (2011). The tonoplast 
copper transporter COPT5 acts as an exporter and is required for interorgan allocation of copper in Arabidopsis thaliana. New Phytol. 192: 393-404.

Pubmed: Author and Title

Google Scholar: Author Only Title Only Author and Title

Kuper, J., Llamas, A, Hecht, H.J., Mendel, R.R., and Schwarz, G. (2004). Structure of the molybdopterin-bound Cnx1G domain links molybdenum and copper metabolism Nature 430: 803-806.

Pubmed: Author and Title

Google Scholar: Author Only Title Only Author and Title

Lanquar, V., Lelièvre, F., Bolte, S., Hamès, C., Acon, C., Neumann, D., Vansuyt, G., Curie, C., Schröder, A, Krämer, U., Barbier-Brygoo, H., and Thomine, S. (2005). Mobilization of vacuolar iron by AtNRAMP3 and AtNRAMP4 is essential for seed germination on low iron.

The EMBO J. 24: 4041-4051.

Pubmed: Author and Title

Google Scholar: Author Only Title Only Author and Title

Lee, N., and Choi, G. (2017). Phytochrome-interacting factor from Arabidopsis to liverwort. Curr Opin Plant Biol. 35: 54-60.

Pubmed: Author and Title

Google Scholar: Author Only Title Only Author and Title

Leivar, P., and Quail, P.H. (2010). PIFs: Pivotal components in a cellular signaling hub. Trends Plant Sci. $16: 19-28$.

Pubmed: Author and Title

Google Scholar: Author Only Title Only Author and Title

Ma, X., Ma. J., Zhai, H., Xin, P., Chu, J., Qiao, Y., and Han, L. (2015). CHR729 is a CHD3 protein that controls seedling development in rice. PLoS One. 10: e0138934.

Pubmed: Author and Title

Google Scholar: Author Only Title Only Author and Title

Mao, Y., Zhang, H., Xu, N., Zhang, B., Gou, F., and Zhu, J.K. (2013). Application of the CRISPR-Cas System for efficient genome engineering in plants. Mol Plant 6: 2008-2011.

Pubmed: Author and Title

Google Scholar: Author Only Title Only Author and Title

Molina-Heredia, F.P., Wastl, J., Navarro, J.A, Bendall, D.S., Hervás, M., Howe, C.J., and De La Rosa, M.A (2003). Photosynthesis: a new function for an old cytochrome? Nature 424: 33-34.

Pubmed: Author and Title

Google Scholar: Author Only Title Only Author and Title

Nambara, E., and Marion-Poll, A (2005). Abscisic acid biosynthesis and catabolism. Annu Rev Plant Biol. 56: $165-185$.

Pubmed: Author and Title

Google Scholar: Author Only Title Only Author and Title

Née, G., Xiang, Y., and Soppe, W.J. (2017). The release of dormancy, a wake-up call for seeds to germinate. Curr Opin Plant Biol. 35: 814.

Pubmed: Author and Title

Google Scholar: Author Only Title Only Author and Title

Nersissian, AM., Immoos, C., Hill, M.G., Hart, P.J., Williams, G., Herrmann, R.G., Valentine, J.S. (1998). Uclacyanins, stellacyanins, and plantacyanins are distinct subfamilies of phytocyanins: plant-specific mononuclear blue copper proteins. Protein Sci. 7: 1915-1929.

Pubmed: Author and Title

Google Scholar: Author Only Title Only Author and Title

Oh, E., Kang, H., Yamaguchi, S., Park, J., Lee, D., Kamiya, Y., Choi G. (2009). Genome-wide analysis of genes targeted by

PHYTOCHROME INTERACTING FACTOR 3-LIKE5 during seed germination in Arabidopsis. Plant Cell 21: 403-419.

Pubmed: Author and Title

Google Scholar: Author Only Title Only Author and Title

Oh, E., Kim, J., Park, E., Kim, J.I., Kang, C., and Choi, G. (2004). PIL5, a phytochrome-interacting basic helix-loop-helix protein, is a key negative regulator of seed germination in Arabidopsis thaliana. Plant Cell 16: 3045-3058.

Pubmed: Author and Title

Google Scholar: Author Only Title Only Author and Title

Oh, E., Yamaguchi, S., Hu, J.H., Yusuke, J., Jung, B., Paik, I., Lee, H.S., Sun, T.P., Kamiya, Y., and Choi, G. (2007). PIL5, a phytochromeinteracting bHLH protein, regulates gibberellin responsiveness by binding directly to the GA and RGA promoters in Arabidopsis seeds. Plant Cell 19: 1192-1208.

Pubmed: $\underline{\text { Author and Title }}$

Google Scholar: Author Only Title Only Author and Title

Oh, E., Yamaguchi, S., Kamiya, Y., Bae, G., Chung, W.I., and Choi, G. (2006). Light activates the degradation of PIL5 protein to promote seed germination through gibberellin in Arabidopsis. Plant J. 47: 124-139.

Pubmed: Author and Title

Google Scholar: Author Only Title Only Author and Title

Pan, J., Huang, D., Guo, Z, Kuang, Z, Zhang, H., Xie, X., Ma, Z, Gao, S., Lerdau, M.T., Chu, C., and Li, L. (2018). Overexpression of 
microRNA408 enhances photosynthesis, growth, and seed yield in diverse plants. J Integr Plant Biol. 60: 323-340.

Pubmed: Author and Title

Google Scholar: Author Only Title Only Author and Title

Paszkiewicz, G., Gualberto, J.M., Benamar, A, Macherel, D., and Logan, D.C. (2017). Arabidopsis seed mitochondria are bioenergetically active immediately upon imbibition and specialize via biogenesis in preparation for autotrophic growth. Plant Cell 29: 109-128.

Pubmed: Author and Title

Google Scholar: Author Only Title Only Author and Title

Peñarrubia, L., Romero, P., Carrió-Seguí, A, Andrés-Bordería, A, Moreno, J., and Sanz, A (2015). Temporal aspects of copper homeostasis and its crosstalk with hormones. Front Plant Sci. 6: 255.

Pubmed: Author and Title

Google Scholar: Author Only Title Only Author and Title

Pfeiffer, A, Shi, H., Tepperman, J.M., Zhang, Y., and Quail, P.H. (2014). Combinatorial complexity in a transcriptionally centered signaling hub in Arabidopsis. Mol Plant 7: 1598-1618.

Pubmed: Author and Title

Google Scholar: Author Only Title Only Author and Title

Roschzttardtz, H., Conéjéro, G., Curie, C., and Mari, S. (2009). Identification of the endodermal vacuole as the iron storage compartment in the Arabidopsis embryo. Plant Physiol. 151: 1329-1338.

Pubmed: Author and Title

Google Scholar: Author Only Title Only Author and Title

Rosenfeld, N., and Alon, U. (2003). Response delays and the structure of transcription networks. J Mol Biol. 329: 645-654.

Pubmed: Author and Title

Google Scholar: Author Only Title Only Author and Title

Ruan, X., Luo, F., Li, D., Zhang, J., Liu, Z, Xu, W., Huang, G., and Li, X. (2011). Cotton BCP genes encoding putative blue copper-binding proteins are functionally expressed in fiber development and involved in response to high-salinity and heavy metal stresses. Physiol

Plant 141: 71-83.

Pubmed: Author and Title

Google Scholar: Author Only Title Only Author and Title

Rydén, L.G., and Hunt, L.T. (1993). Evolution of protein complexity: the blue copper-containing oxidases and related proteins. J Mol Evol. 36: 41-66.

Pubmed: Author and Title

Google Scholar: Author Only Title Only Author and Title

Sakuraba, Y., Kanno, S., Mabuchi, A, Monda, K., Iba, K., and Yanagisawa, S. (2018). A phytochrome-B-mediated regulatory mechanism of phosphorus acquisition. Nat Plants 4: 1089-1101.

Pubmed: Author and Title

Google Scholar: Author Only Title Only Author and Title

Schulten, A, Bytomski, L., Quintana, J., Bernal, M., and Krämer, U. (2019). Do Arabidopsis Squamosa promoter binding Protein-Like genes act together in plant acclimation to copper or zinc deficiency? Plant Direct 3: e00150.

Pubmed: Author and Title

Google Scholar: Author Only Title Only Author and Title

Seo, M., Nambara, E., Choi, G., and Yamaguchi, S. (2009). Interaction of light and hormone signals in germinating seeds. Plant Mol Biol. 69: 463-472.

Pubmed: Author and Title

Google Scholar: Author Only Title Only Author and Title

Seo, M., Peeters, AJ., Koiwai, H., Oritani, T., Marion-Poll, A, Zeevaart, J.A, Koornneef, M., Kamiya, Y., and Koshiba T. (2000). The Arabidopsis aldehyde oxidase 3 (AAO3) gene product catalyzes the final step in abscisic acid biosynthesis in leaves. Proc Natl Acad Sci U S A 97: 12908-12913.

Pubmed: Author and Title

Google Scholar: Author Only Title Only Author and Title

Shen, H., Zhu, L., Castillon, A, Majee, M., Downie, B., and Huq, E. (2008). Light-induced phosphorylation and degradation of the negative regulator PHYTOCHROME-INTERACTING FACTOR1 from Arabidopsis depend upon its direct physical interactions with photoactivated phytochromes. Plant Cell 20: 1586-1602.

Pubmed: Author and Title

Google Scholar: Author Only Title Only Author and Title

Shi, H., Lyu, M., Luo, Y., Liu, S., Li, Y., He, H., Wei, N., Deng, X.W., and Zhong, S. (2018). Genome-wide regulation of light-controlled seedling morphogenesis by three families of transcription factors. Proc Natl Acad Sci U S A 115: 6482-6487.

Pubmed: Author and Title

Google Scholar: Author Only Title Only Author and Title

Shi, H., Wang, X., Mo, X., Tang, C., Zhong, S., Deng, X.W. (2015) Arabidopsis DET1 degrades HFR1 but stabilizes PIF1 to precisely regulate seed germination. Proc Natl Acad Sci U S A 112: 3817-3822. 
Pubmed: Author and Title

Google Scholar: Author Only Title Only Author and Title

Shi, H., Zhong, S., Mo, X., Liu, N., Nezames, C.D., Deng, X.W. (2013). HFR1 sequesters PIF1 to govern the transcriptional network underlying light-initiated seed germination in Arabidopsis. Plant Cell 25: 3770-3784.

Pubmed: Author and Title

Google Scholar: Author Only Title Only Author and Title

Shoval, O., and Alon, U. (2010). SnapShot: network motifs. Cell 143: 326-e1.

Pubmed: Author and Title

Google Scholar: Author Only Title Only Author and Title

Shu, K., Liu, X.D., Xie, Q., and He, ZH. (2016). Two faces of one seed: hormonal regulation of dormancy and germination. Mol Plant 9:

34-45.

Pubmed: Author and Title

Google Scholar: Author Only Title Only Author and Title

Sun, T.P., and Kamiya, Y. (1997). Regulation and cellular localization of ent-kaurene synthesis. Physiol Plant. 101: $701-708$.

Pubmed: Author and Title

Google Scholar: Author Only Title Only Author and Title

Sun, Y, Wu, Z, Wang, Y, Yang, J, Wei, G, and Chou, M. (2019). Identification of phytocyanin gene family in legume plants and their involvement in nodulation of Medicago truncatula. Plant Cell Physiol. 60: 900-915.

Pubmed: Author and Title

Google Scholar: Author Only Title Only Author and Title

Toledo-Ortiz, G., Johansson, H., Lee, K.P., Bou-Torrent, J., Stewart, K., Steel, G., Rodríguez-Concepción, M., and Halliday, K.J. (2014). The HY5-PIF regulatory module coordinates light and temperature control of photosynthetic gene transcription. PLoS Genet. 10: e1004416.

Pubmed: Author and Title

Google Scholar: Author Only Title Only Author and Title

Wang, K., and Frame, B. (2009). Biolistic gun-mediated maize genetic transformation. Methods Mol Biol. 526: 29-45.

Pubmed: Author and Title

Google Scholar: Author Only Title Only Author and Title

Weigel, M., Varotto, C., Pesaresi, P., Finazzi, G., Rappaport, F., Salamini, F., and Leister, D. (2003). Plastocyanin is indispensable for photosynthetic electron flow in Arabidopsis thaliana. J. Biol Chem 278: 31286-31289.

Pubmed: Author and Title

Google Scholar: Author Only Title Only Author and Title

Winter, D., Vinegar, B., Nahal, H., Ammar, R., Wilson, G.V., and Provart, N.J. (2007). An "Electronic Fluorescent Pictograph" browser for exploring and analyzing large-scale biological data sets. PLoS One. 8: e718.

Pubmed: Author and Title

Google Scholar: Author Only Title Only Author and Title

Yamaguchi, S. (2008). Gibberellin metabolism and its regulation. Annu Rev Plant Biol. 59: 225-251.

Pubmed: Author and Title

Google Scholar: Author Only Title Only Author and Title

Ye, N., Li, H., Zhu, G., Liu, Y., Liu, R., Xu, W., Jing, Y., Peng, X., and Zhang, J. (2014). Copper suppresses abscisic acid catabolism and catalase activity, and inhibits seed germination of rice. Plant Cell Physiol. 55: 2008-2016.

Pubmed: Author and Title

Google Scholar: Author Only Title Only Author and Title

Yoo, S.D., Cho, Y.H., and Sheen, J. (2007). Arabidopsis mesophyll protoplasts: a versatile cell system for transient gene expression analysis. Nat Protoc. 2: 1565-1572.

Pubmed: Author and Title

Google Scholar: Author Only Title Only Author and Title

Zhang, H., and Li, L. (2013). SQUAMOSA promoter binding protein-like7 regulated microRNA408 is required for vegetative development in Arabidopsis. Plant J. 74: 98-109.

Pubmed: Author and Title

Google Scholar: Author Only Title Only Author and Title

Zhang, H., Zhao, X., Li, J., Cai, H., Deng, X.W., and Li, L. (2014). MicroRNA408 is critical for the HY5-SPL7 gene network that mediates the coordinated response to light and copper. Plant Cell 26: 4933-4953.

Pubmed: Author and Title

Google Scholar: Author Only Title Only Author and Title

Zhong, S., Shi, H., Xue, C., Wei, N., Guo, H., and Deng, X.W. (2014). Ethylene-orchestrated circuitry coordinates a seedling's response to soil cover and etiolated growth. Proc Natl Acad Sci U S A 111: 3913-3920.

Pubmed: Author and Title

Google Scholar: Author Only Title Only Author and Title 
Zhong, S., Zhao, M., Shi, T., Shi, H., An, F., Zhao, Q., and Guo, H. (2009). EIN3/EIL1 cooperate with PIF1 to prevent photo-oxidation and to promote greening of Arabidopsis seedlings. Proc Natl Acad Sci U S A 106: 21431-21436.

Pubmed: Author and Title

Google Scholar: Author Only Title Only Author and Title

Zhou, M.G., Gu, L.F., Li, P.C., Song, X.W., Wei, L.Y., Chen, ZY., and Cao, X.F. (2010). Degradome sequencing reveals endogenous small RNA targets in rice (Oryza sativa L. ssp. indica). Front Biol. 5: 67-90.

Pubmed: Author and Title

Google Scholar: Author Only Title Only Author and Title

Zhuang, Y., Zuo, D., Tao, Y., Cai, H., and Li, L. (2020). Laccase3-based extracellular domain provides possible positional information for directing Casparian strip formation in Arabidopsis. Proc Natl Acad Sci U S A 117: 15400-15402.

Pubmed: Author and Title

Google Scholar: Author Only Title Only Author and Title

Zuo, J., Niu, Q.W., and Chua, N.H. (2000). An estrogen receptor-based transactivator XVE mediates highly inducible gene expression in transgenic plants. Plant J. 24: 265-273.

Pubmed: Author and Title

Google Scholar: Author Only Title Only Author and Title 